\title{
CORRELACIONES A PARTIR DE LA INTENSIDAD DE ARIAS PARA DATOS ACELEROGRÁFICOS DE COSTA RICA
}

\author{
Víctor Schmidt Díaz \\ Universidad Politécnica de Cataluña, Instituto Geológico de Cataluña, Balmes \\ 209-211, E-08006, Barcelona, España \\ Laboratorio de Ingeniería Sísmica, Universidad de Costa Rica Nivel 3-A Facultad \\ de Ingeniería, Universidad de Costa Rica, 2060 San Pedro de Montes de Oca \\ vschmidt@eic.ucr.ac.cr
}

(Recibido 31/08/07; aceptado 03/05/08)

\begin{abstract}
This research purposes the use of the Arias Intensity (IA) index as an indicator of the strength of an earthquake, which can be correlated with other seismic parameters as the moment magnitude (Mw), hypocentral distance (D), peak ground acceleration (PGA), geotechnical conditions of the stations (S) and the Modified Mercalli Intensity scale (IMM) assigned to each site after the occurrence of an earthquake. The original database consists in 1018 free field strong motion records obtained between 1983 and 2006 by the Earthquake Engineering Laboratory of the University of Costa Rica and the Costa Rican Institute of Electricity. After defining some selection criteria, the amount of records diminished to 734 which come from 320 felt earthquakes. The correlations obtained after several tests are: $\mathrm{IA}=8 \mathrm{E}-6 *(\mathrm{PGA})^{1,9956}, \mathrm{r}^{2}=0,923, \mathrm{Ln}(\mathrm{IA})=-13,799+2,685^{*} \mathrm{Mw}-1,611 * \operatorname{Ln}(\mathrm{D})-0,0034 * \mathrm{D}+0,945 * \mathrm{~S}, \mathrm{r}^{2}=0,71 . \mathrm{IMM}=$ $0,5719 * \operatorname{Ln}(\mathrm{IA})+7,1952, \mathrm{r}^{2}=0,9905$. Results show high correlation coefficient for every regression, as well as similar behavior in some cases considering the ones proposed by authors using different worldwide databases.

Keywords: Arias and Mercalli intensities, regressions, accelerograms, Costa Rica.

RESUMEN: Se plantea el uso de la Intensidad de Arias (IA), como un indicador de la fuerza del sismo y que puede ser correlacionado con otros parámetros, como la magnitud de momento (Mw), la distancia hipocentral (D), la aceleración pico del suelo (PGA), las condiciones geotécnicas de la estación de registro (S) y la Intensidad Mercalli Modificada (IMM) asignada a este sitio luego de la ocurrencia de un sismo. El banco de datos utilizado consta de 1018 registros acelerográficos de campo libre obtenidos entre 1983 y el 2006 por el Laboratorio de Ingeniería Sísmica de la Universidad de Costa Rica y por el Instituto Costarricense de Electricidad. Luego de definir una serie de criterios de selección, el grupo de datos se reduce a 734 registros provenientes de 320 sismos sentidos. Después de una serie de pruebas, se obtuvieron las siguientes relaciones:
\end{abstract}

SCHMIDT, V., 2008: Correlaciones a partir de la Intensidad de Arias para datos acelerográficos de Costa Rica. - Rev. Geol. Amér. Central, 38: 95-117. 
$\mathrm{IA}=8 \mathrm{E}-6 *(\mathrm{PGA})^{1,9956}, \mathrm{r}^{2}=0,923,\left(\mathrm{IA}\right.$ en $\mathrm{m} / \mathrm{s}$ y PGA en $\left.\mathrm{cm} / \mathrm{s}^{2}\right), \operatorname{Ln}(\mathrm{IA})=-13,799+2,685 * \mathrm{Mw}-1,611 * \operatorname{Ln}(\mathrm{D})-$ $0,0034 * \mathrm{D}+0,945 * \mathrm{~S}, \mathrm{r}^{2}=0,71$ (IA en $\mathrm{m} / \mathrm{s}$ y $\mathrm{D}$ en $\mathrm{km}$, Mw y S son adimensionales), IMM $=0,5719 * \operatorname{Ln}(\mathrm{IA})+7,1952$, $\mathrm{r}^{2}=0,9905$ (IMM es adimensional e IA en $\mathrm{m} / \mathrm{s}$ ). Los resultados muestran altos coeficientes de correlación, así como similitud en algunos casos con ecuaciones propuestas por otros autores, obtenidas a partir de datos de otras partes del mundo.

Palabras clave: Intensidades de Arias y Mercalli, regresiones, acelerogramas, Costa Rica.

\section{INTRODUCCIÓN}

En términos generales, después de la ocurrencia de un sismo sentido, es común que en medios periodísticos se reporten los valores de la intensidad macrosísmica asociados con ese evento, provenientes de las distintas comunidades donde el fenómeno fue percibido. Esto suele dar una idea del efecto que el movimiento tuvo, tanto en la población en general como en las obras civiles existentes en la zona. Sin embargo, hasta el día de hoy la intensidad macrosísmica resulta ser un parámetro de difícil asignación, ya que no proviene de una medida instrumental, sino más bien se basa en la forma en que las personas perciben el movimiento en un sitio en concreto, así como en efectos que estas observan a su alrededor. De acuerdo con la definición de la escala Mercalli Modificada (Wood \& Newman, 1931) que es la utilizada comúnmente en Costa Rica (Sauter, 1989), la asignación de un grado correcto resulta ser especialmente crítica para grados de intensidad VI o inferiores, ya que en ese rango de valores no existe daño estructural aparente, por lo que el reporte de intensidades se basa en observaciones y percepciones subjetivas.

Es por esta razón que investigadores alrededor del mundo han propuesto indicadores de la fuerza con que un sismo sacude un sitio específico que se basan en medidas instrumentales. Arias (1970) propuso un indicador por medio del cual se obtiene una medida del contenido energético de un registro sísmico, basado en cada valor de aceleración registrado a lo largo del tiempo, así como en la duración total del movimiento, por lo que resulta ser un índice muy completo o "robusto". Además, a partir de este parámetro ha sido posible definir umbrales referentes al desempeño y al daño estructural, al potencial de licuefacción y a la estabilidad de taludes, entre otros (Travasarou et al., 2003).

Este indicador se relaciona con la aceleración pico del suelo, estudia el proceso de atenuación que sufre en función de la magnitud, la distancia hipocentral y las condiciones del suelo en el sitio de registro y finalmente es correlacionado con los reportes existentes de Intensidad Mercalli Modificada para algunos de los sismos más importantes sucedidos en Costa Rica durante los últimos 23 años.

Este trabajo pretende aportar correlaciones que permitan considerar la Intensidad de Arias como un indicador más confiable de la fuerza con que un sismo sacude un sitio específico, a partir de una selección detallada de datos y registros acelerográficos obtenidos en Costa Rica desde 1983 hasta el 2006 por las redes del Laboratorio de Ingeniería Sísmica (LIS) y el Instituto Costarricense de Electricidad (ICE).

\section{DEFINICIONES TEÓRICAS Y CONSIDERACIONES PRELIMINARES}

Los conceptos y bases teóricas a partir de los cuales se lleva a cabo el análisis son:

\section{Intensidad de Arias (IA)}

Propuesta por el investigador chileno Arturo Arias en 1970, se define como la energía por unidad de peso disipada por una familia de osciladores de un grado de libertad, cuyas frecuencias están comprendidas en el rango de $(0,+\infty)$, para un sismo y amortiguamiento dados. La deducción de 
su expresión matemática considera como modelo estructural el oscilador de un grado de libertad con amortiguamiento viscoso. La expresión simplificada es:

$$
I_{A}=\frac{\pi}{2 g} \int_{0}^{t_{0}} \ddot{u}_{g}^{2}(\tau) d \tau
$$

Donde $\tau$ es una variable temporal, $\ddot{u}_{\mathrm{g}}$ es la aceleración del suelo durante el movimiento sísmico y $\mathrm{t}_{0}$ es la duración total del movimiento, cuya definición queda supeditada a la duración de la fase fuerte, a la total del registro acelerográfico o al criterio del investigador. En este caso se considerará la duración total del registro.

\section{Intensidad Mercalli Modificada (IMM)}

La intensidad IMM es una medida del movimiento del suelo causado por la ocurrencia de un evento sísmico y del grado con que la vibración es sentida y registrada en una determinada localidad. Depende del sitio de observación, generalmente es mayor en el área epicentral y disminuye con la distancia respecto al epicentro. La escala IMM se extiende del grado I al XII. La asignación de los grados de I a VI se hace a partir de la percepción individual, la de los grados VII a X depende de la severidad de los daños causados a las edificaciones y finamente la asignación de los grados XI y XII se basa en efectos claramente destructivos y cambios geológicos producidos por el terremoto (Wood \& Newman, 1931). Cada grado tiene una descripción más o menos detallada y esta es la escala comúnmente utilizada en Costa Rica.

\section{Aceleración pico del suelo (PGA)}

Se define como la aceleración pico del suelo a la máxima aceleración en valor absoluto en toda la historia temporal de un acelerograma, obtenido cuando ocurre un sismo. Dado que la mayoría de registros acelerográficos cuenta con tres componentes, normalmente se leen tres PGA: el longitudinal, que suele coincidir con la dirección N-S, el vertical y el transversal, que corresponde con la dirección E-W. En este estudio solamente se trabajará con las componentes en el plano horizontal, o sea, la longitudinal y la transversal al instrumento.

\section{Magnitud de momento $\left(M_{w}\right)$}

Representa una escala de magnitud, que para efectos de esta investigación se relaciona con las demás escalas siguiendo las correlaciones propuestas por Rojas et al., (1993) a partir de datos de América Central, de la siguiente forma:

$$
\begin{aligned}
& M_{S}=-4,165+1,783^{*} M_{D} \\
& M_{W}=2,251+0,655^{*} M_{S}
\end{aligned}
$$

$$
\text { Y combinando (2) y (3) }
$$$$
M_{W}=-0,447+1,168 * M_{D}
$$

Donde $\mathrm{M}_{\mathrm{w}}$ es la magnitud de momento, $\mathrm{M}_{\mathrm{D}}$ es la magnitud local y $\mathrm{M}_{\mathrm{S}}$ es la obtenida a partir de ondas superficiales. Se utilizará la magnitud de momento debido a que es una medida muy estable del tamaño del terremoto, que físicamente define mejor a la fuente y da una estimación más exacta de la energía liberada (Boore \& Joyner, 1982; Sauter, 1989).

\section{Tipo de suelo}

Para definir las condiciones del sitio donde se ubican las estaciones utilizadas en este trabajo, se definieron cuatro tipos que cumplen aproximadamente con las características descritas por el Código Sísmico de Costa Rica 2002 (CFIA, 2003). No se contó con resultados de pruebas 
geofísicas ni geotécnicas para esta clasificación, sino que se hizo a partir de observaciones de campo y del estudio de mapas geológicos e información geotécnica disponible. Los tipos de suelo según la referencia citada son:

- Sitio tipo $S_{1}$ : Un perfil de suelo con un material semejante a roca, caracterizado por una velocidad de onda cortante superior a 760 $\mathrm{m} / \mathrm{s}$ o por otros medios adecuados de clasificación; o bien, condiciones de suelo rígido o denso, donde la profundidad del suelo es menor de $50 \mathrm{~m}$. En adelante a este tipo de sitio se le denominará roca.

- Sitio tipo $S_{2}$ : Un perfil de suelo con condiciones predominantes de medianamente denso a denso o de medianamente rígido a rígido, cuya profundidad excede los $50 \mathrm{~m}$. En adelante a esta condición se le denominará suelo firme.

- Sitio tipo $\mathrm{S}_{3}$ : Un perfil de suelo con más de $6 \mathrm{~m}$ de arcilla de consistencia de suave a medianamente rígida o de suelos no cohesivos de poca o mediana densidad. No incluye perfiles de más de $12 \mathrm{~m}$ de arcilla suave. En adelante se considerará como suelo blando.

- Sitio tipo $\mathrm{S}_{4}$ : Un perfil de suelo caracterizado por una velocidad de onda cortante menor que $150 \mathrm{~m} / \mathrm{s}$ o con más de $12 \mathrm{~m}$ de arcilla suave. Se le denominará suelo muy blando.

\section{Distancia hipocentral}

Es la hipotenusa del triángulo rectángulo cuyos catetos son la distancia epicentral (distancia entre la proyección del foco en la superficie terrestre y un sitio específico, que en este caso será la ubicación de la estación acelerográfica) y la profundidad hipocentral (distancia entre el foco o hipocentro y su proyección en la superficie).

\section{Regresión empírica para la intensidad de Arias}

La predicción empírica del movimiento del suelo parte de la relación existente entre una variable dependiente con otras independientes. Una expresión ampliamente aceptada para relacionar estos tipos de variables es la siguiente (Boore \& Joyner, 1982):

$$
Y=b_{1} e^{b_{2} M}\left[e^{b_{3} D} / D^{b_{4}} \oint^{b_{5} S} e^{b_{6} P}\right.
$$

Donde $\mathrm{Y}$ es la variable dependiente que suele ser considerada como la aceleración pico del suelo, la aceleración espectral o la velocidad pico del suelo. En este trabajo, será tomada la Intensidad de Arias como dependiente, tal y como han hecho otros autores (Travasarou et al., 2003).

Las variables independientes son: la magnitud $\mathrm{M}$, que en este caso será utilizada la $\mathrm{M}_{\mathrm{w}}, \mathrm{D}$ que es una medida de distancia y en este trabajo se considerará como la hipocentral, aún cuando sería más conveniente trabajar con la distancia a la zona de ruptura, pero no está disponible en la mayoría de los caos, $\mathrm{S}$ que es una variable binaria que representa la geología local del sitio, $\mathrm{P}$ que está asociada a la incertidumbre en la predicción (0 y 1 para los percentiles 50 y 84, respectivamente). Finalmente $b_{n}$ son los coeficientes a determinar en el análisis de regresión.

Aplicando logaritmos a ambos lados de la ecuación (5), queda en su forma linearizada como:

$\ln Y=c_{0}+c_{m} M-c_{L D} \ln D-c_{D} D+c_{S} S+c_{p} P$

Con esta formal lineal, los términos c que corresponden a los $b_{n}$ en la ecuación (5), serán los que se determinarán por medio de la inversión entre las distintas variables independientes $(\mathrm{M}$, D, S y P) respecto a la dependiente Y. Los coeficientes tienen el siguiente significado físico: $\mathrm{c}_{0}$ se refiere a la fuente generadora del sismo, $\mathrm{c}_{\mathrm{m}}$ es el coeficiente de escala de la magnitud, $\mathrm{c}_{\mathrm{LD}}$ está relacionado con la propagación geométrica de las ondas, $\mathrm{c}_{\mathrm{D}}$ corresponde a la atenuación anelástica, $\mathrm{c}_{\mathrm{S}}$ con la condición de suelo. A $\mathrm{c}_{\mathrm{P}}$ se le denomina tradicionalmente $\Sigma$ (épsilon), que representa el número de desviaciones estándar que se toman en la predicción del movimiento para una cierta magnitud y distancia. En el modelo que se propone, este término no fue considerado debido a que, al ser limitada la cantidad de datos disponibles, cada término agregado a la ecuación (6) puede provocar su inestabilidad y generar predicciones muy lejanas a la realidad. Por esta razón, la fiabilidad del modelo se basa en el coeficiente de determinación $\left(\mathrm{r}^{2}\right)$ y en el cálculo de residuos, 
que se detallará más adelante. Precisamente, de acuerdo con Boore \& Joyner (1982), es criterio del investigador trabajar con parte o la totalidad de los términos de la ecuación (6), de acuerdo con la disponibilidad y fiabilidad de los datos, entre otros factores.

Por otro lado, el término correspondiente al tipo de suelo se tratará de la siguiente forma: debido a que los datos referentes a la condición de roca son escasos (ver el capítulo siguiente), y a que esta condición es difícil de definir para algunos de los sitios existentes debido a la ausencia de pruebas geofísicas que permitan la estimación de las velocidades de onda cortante de los estratos, se tomó la decisión de agrupar las condiciones: roca y suelo firme como una única categoría denominada "firme" (valor $\mathrm{S}$ de 0 ), y la correspondiente a suelo blando y muy blando se trabajó como "blando" (valor S de 1).

\section{EL BANCO DE DATOS Y EL PROCESO DE SELECCIÓN DE LOS REGISTROS}

Los 1018 registros acelerográficos considerados en este estudio provienen de estaciones localizadas en campo libre (en la mayoría de los casos ubicadas en estructuras de uno o dos pisos para evitar el efecto de la interacción suelo-estructura) dentro del territorio costarricense, obtenidos en un periodo de tiempo de 23 años (de 1983 al 2006), por las redes acelerográficas del Laboratorio de Ingeniería Sísmica (LIS) y el Instituto Costarricense de Electricidad (ICE). Los acelerogramas corresponden a 413 eventos sísmicos en un rango de magnitudes $M_{w}$ entre 2,2 y 7,7 y un rango de distancias hipocentrales de 3 a $747 \mathrm{~km}$.

El cuadro 1 muestra la distribución de datos según la red de acelerógrafos que los registró (LIS o ICE), el tipo de fuente (subducción o fallamiento local) y el tipo de suelo donde se ubican las estaciones (roca, firme o blando). Se observa que la mayor cantidad de datos los aporta el LIS, y la mayor parte corresponde con suelo blando y la menor a roca. Respecto al origen, los porcentajes correspondientes a subducción y a fallamiento local son bastante semejantes.

Se muestra la distribución de los datos según la relación Magnitud (Mw) vs. distancia hipocentral en la fig. 1. Se observa una clara tendencia a que los datos se ubiquen a lo largo de una franja diagonal, siendo escasos para magnitudes bajas y distancias lejanas, pero sobre todo se cuenta con pocos datos para magnitudes altas y distancias cortas, tal y como suele suceder cuando se analizan datos provenientes de pocas redes acelerográficas. Sin embargo, se considera que es una distribución aceptable para llevar a cabo el análisis que se propone, en comparación con estudios semejantes hechos en otras partes del mundo. En todo caso, lo recomendable sería que estos resultados sean revisados y replanteados cuando se cuente con una cantidad significativa de registros nuevos.

La figura 2 presenta la ubicación de las estaciones y de los epicentros con respecto a Costa Rica y su vecindad. Hay una clara tendencia a que los epicentros se concentren a lo largo de la costa del Pacífico Central, así como hacia el centro geográfico del país donde se ubica el Valle Central, que es donde se concentra la mayor cantidad de población, bienes y servicios. Para datos específicos de cada estación, así como de cada instrumento y acelerograma, se sugiere consultar el cibersitio www.fing.ucr.ac.cr/ lis/

Los instrumentos de registro utilizados corresponden a diversos modelos de la marca Kinemetrics Inc.: SMA-1 (analógico), SSA-1 y 2, ETNA, K2 (digitales) y en menor proporción, convertidores analógico-digital tipo QDR.

A todos los registros se les aplicó un filtro pasa-banda tipo Ormsby en un rango de frecuencias de corte de $0,8 \mathrm{~Hz}$ a $23 \mathrm{~Hz}$ para los analógicos y de 0,12 a $47 \mathrm{~Hz}$ para los digitales. También se les aplicó corrección por línea base utilizando el programa suministrado por el mismo fabricante

Cuadro 1

Distribución de los datos considerados en este estudio

\begin{tabular}{ccc}
\hline Total & 1018 & $\%$ \\
\hline Datos LIS & 971 & 95 \\
Datos ICE & 47 & 5 \\
Datos LIS subducción & 543 & 53 \\
Datos LIS fallamiento local & 475 & 47 \\
Roca & 179 & 17 \\
Suelo firme & 334 & 33 \\
Suelo blando y muy blando & 505 & 50 \\
\hline
\end{tabular}




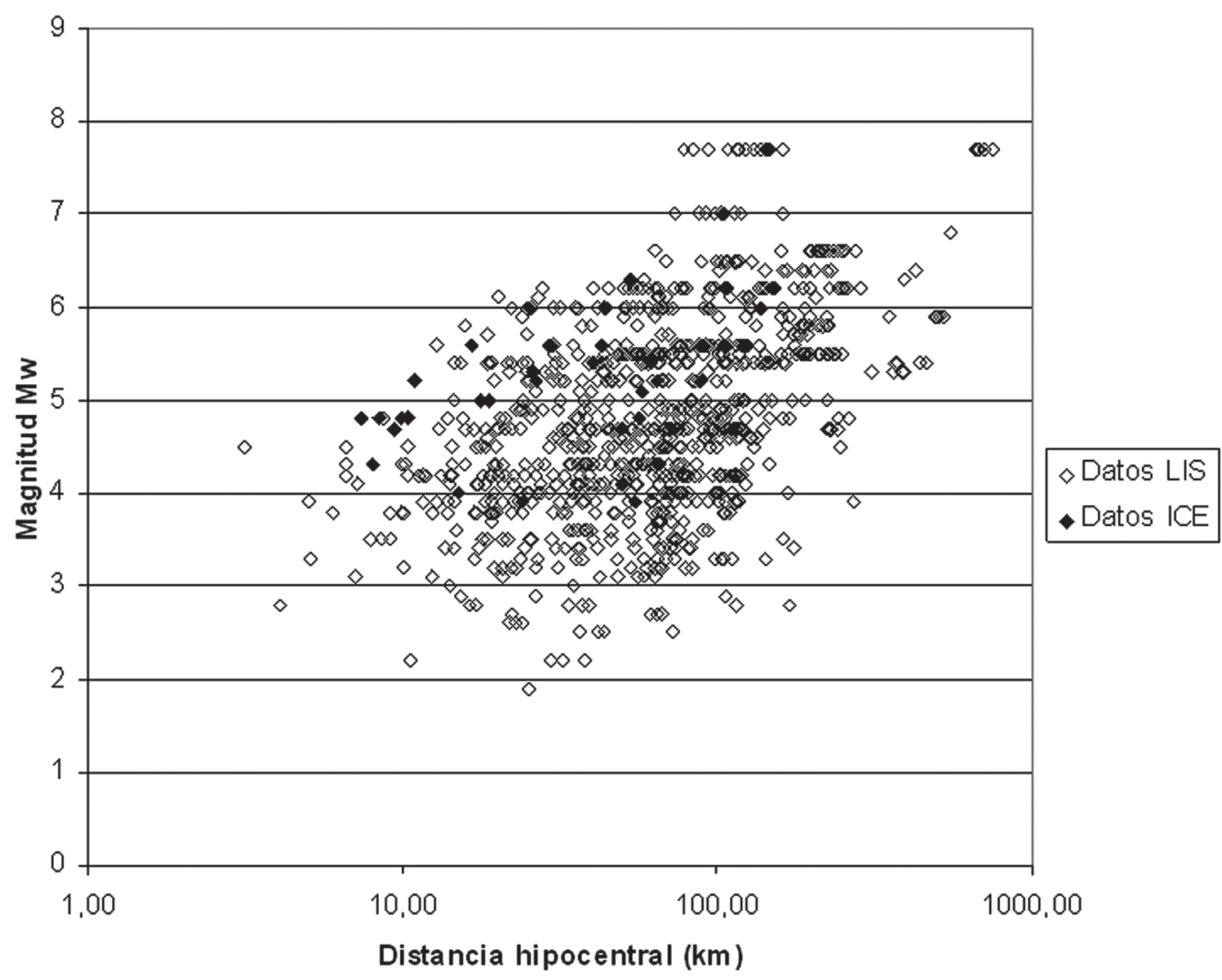

Fig. 1: Distribución de los datos utilizados en el estudio considerando magnitud y distancia hipocentral.

de los instrumentos. Se fijó una frecuencia pasaalta de $0,8 \mathrm{~Hz}$ para los registros que provienen de equipo analógico, debido a que son instrumentos con muchas limitaciones de resolución, sobretodo cuando registran PGA bajos, situación común en los acelerogramas utilizados en esta investigación. Por otro lado, es una frecuencia de filtro recomendada por el fabricante y por numerosos usuarios para este tipo de instrumentos. Se puede tener como consecuencia IA ligeramente más bajos respecto a los no filtrados; sin embargo, los registros analógicos no representan la mayoría de los acelerogramas tomados en cuenta.

Debido a que fueron considerados registros desde el año 1983, época en la cual predominaban los acelerógrafos analógicos, fue necesario hacer una selección minuciosa de los registros a utilizar, dado que algunos de los más antiguos presentaban problemas de digitalización o bien, niveles muy bajos de aceleración que no sobre- pasaban el ancho de la línea de la traza. Por otro lado, algunos registros digitales presentaron niveles de ruido muy altos con respecto al de la señal o en otros casos, presentaban picos inconsistentes con respecto a la forma de onda esperada. Se muestran algunos ejemplos de registros considerados como aceptables y del tipo correspondiente a los eliminados (Fig. 3).

De los 1018 registros originales, después de analizar todas las formas de onda (registros de aceleración en el tiempo), se desecharon 284 (28\% del total), para dejar un grupo de registros apto para el análisis de 734 (72\% del grupo original de datos), correspondiente con 320 sismos percibidos.

Las Figs. 4 y 5 muestran las distribuciones del número de registros vs. magnitud y distancia hipocentral para los datos que fueron seleccionados. Se observa que la mayor cantidad de datos se ubica en un rango de magnitudes entre 4 y 5 


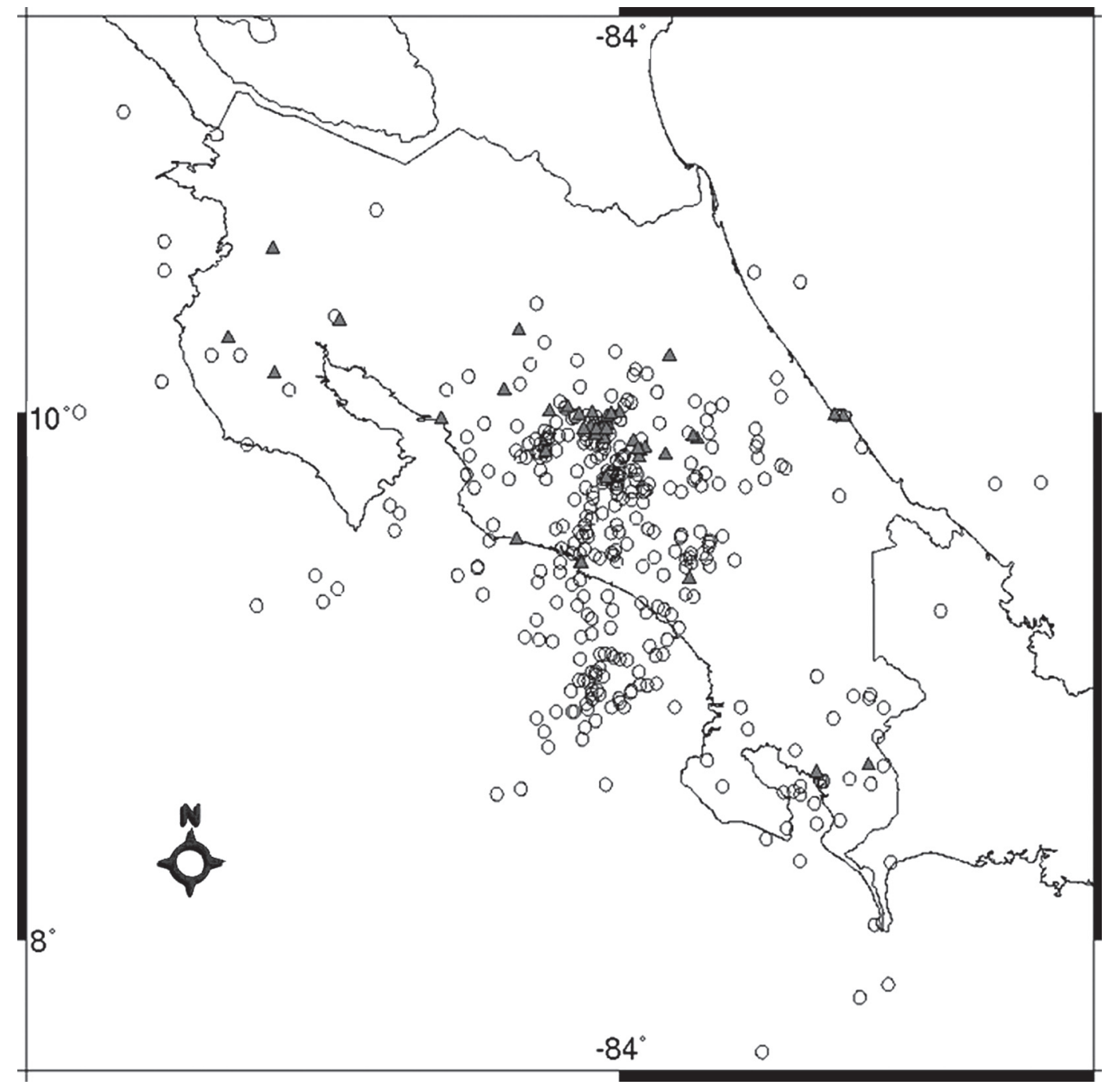

Fig. 2: Distribución espacial de los epicentros (círculos) y de las estaciones (triángulos)

y que las distancias hipocentrales predominantes van de 20 a $40 \mathrm{~km}$, siendo también frecuentes de 40 a $80 \mathrm{~km}$. También se muestra la distribución de los datos utilizados según su relación magnitud Mw vs. distancia hipocentral (Fig. 6), donde se observa la ausencia de datos de magnitudes bajas a distancias largas y de magnitudes altas a distancias cortas.

Es conveniente recalcar que prácticamente no existen estaciones ubicadas en condición de roca sin algún grado de meteorización, por lo que se decidió agrupar a la condición de roca y suelo firme como una sola categoría. Por lo tanto, la condición de suelo en los registros queda definida por dos categorías: suelo firme y suelo blando, tal y como se dijo antes.

\section{RESULTADOS Y DISCUSIÓN}

Con base en los resultados obtenidos, se relaciona la Intensidad de Arias (IA) con la aceleración pico del suelo (PGA), se analiza su atenuación respecto a parámetros sismológicos y geotécnicos y finalmente, se correlaciona con la Intensidad Mercalli Modificada (IMM). 

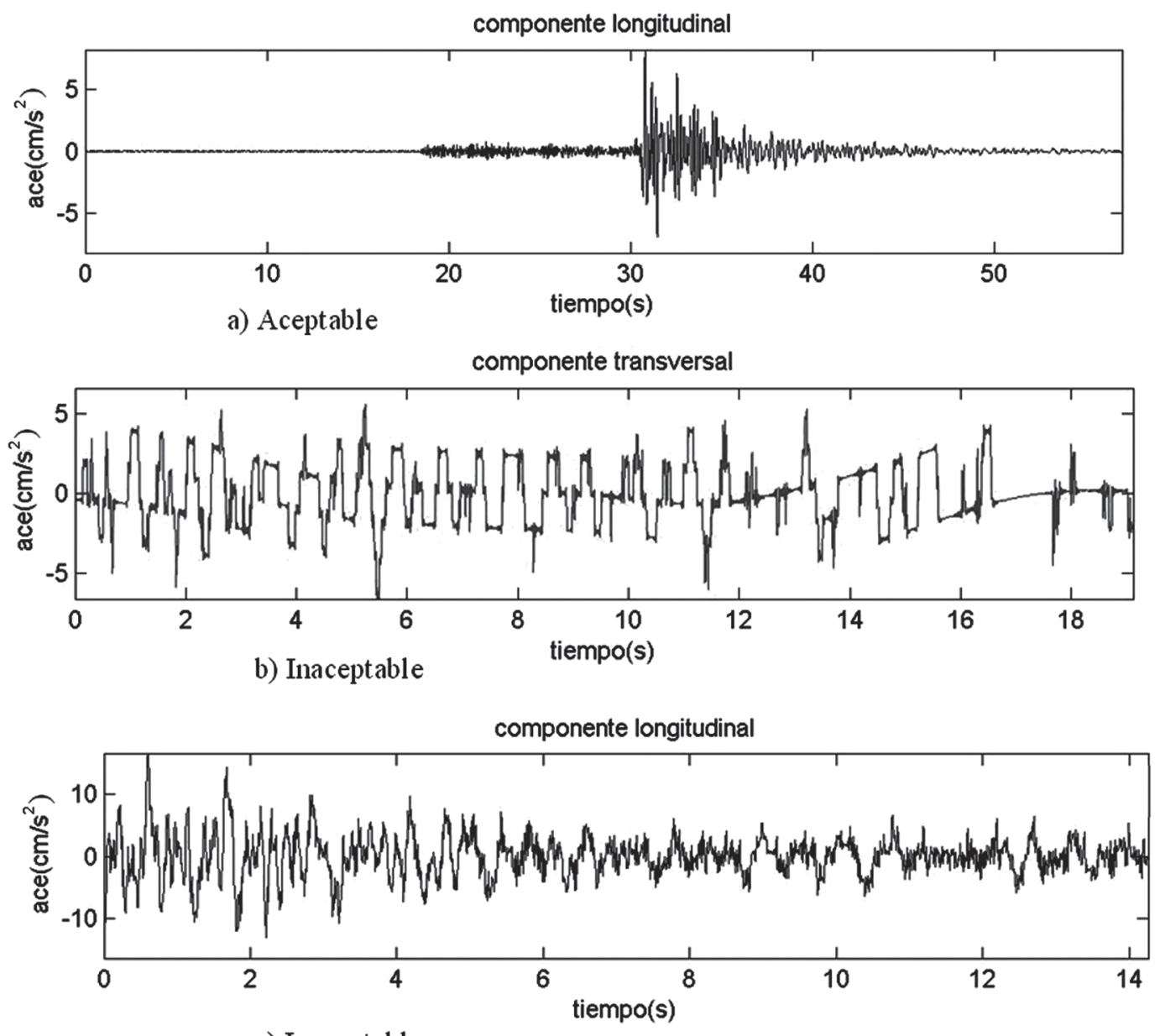

c) Inaceptable

Fig. 3: Distintos acelerogramas: a) Tipo de acelerograma considerado como aceptable. b) Acelerograma desechado debido a que proviene de un instrumento analógico y con amplitudes muy bajas. c) Acelerograma digital considerado como inaceptable debido a su elevado nivel de ruido respecto a las amplitudes de la señal y por estar incompleto.

\section{Correlaciones entre IA y PGA}

Aún cuando la PGA es un único valor que se lee en la historia tiempo-aceleración de cada componente de un registro acelerográfico, y que la IA toma en cuenta tanto la duración total como todos los valores de aceleración contenidos en el acelerograma, se plantea en este trabajo establecer correlaciones entre ambos parámetros de modo que, en caso de obtener resultados satisfactorios, será posible estimar la IA de forma inmediata al conocer la PGA correspondiente a un registro, sin necesidad de contar con el acelerograma completo, situación que suele ser bastante común.
Las Figs. 7a y $7 \mathrm{~b}$ muestran las correlaciones obtenidas. En la figura 7a se presenta la línea de mejor ajuste considerando solamente la IA máxima de las dos componentes horizontales por registro (734 en total). El ajuste es bastante bueno, con un coeficiente de correlación $\mathrm{r}^{2}=0,9215$. Se llevó a cabo el mismo procedimiento, pero con ambas componentes horizontales del acelerograma (1468 datos; Fig. 7b), por lo tanto, cada registro aporta dos valores de IA. Obsérvese que la correlación mejora ligeramente.

Se puede notar también que la mayor cantidad de datos se concentran alrededor de $\mathrm{PGA}=10$ gals $\left(1 \mathrm{gal}=1 \mathrm{~cm} / \mathrm{s}^{2}\right)$, siendo más escasos a partir 


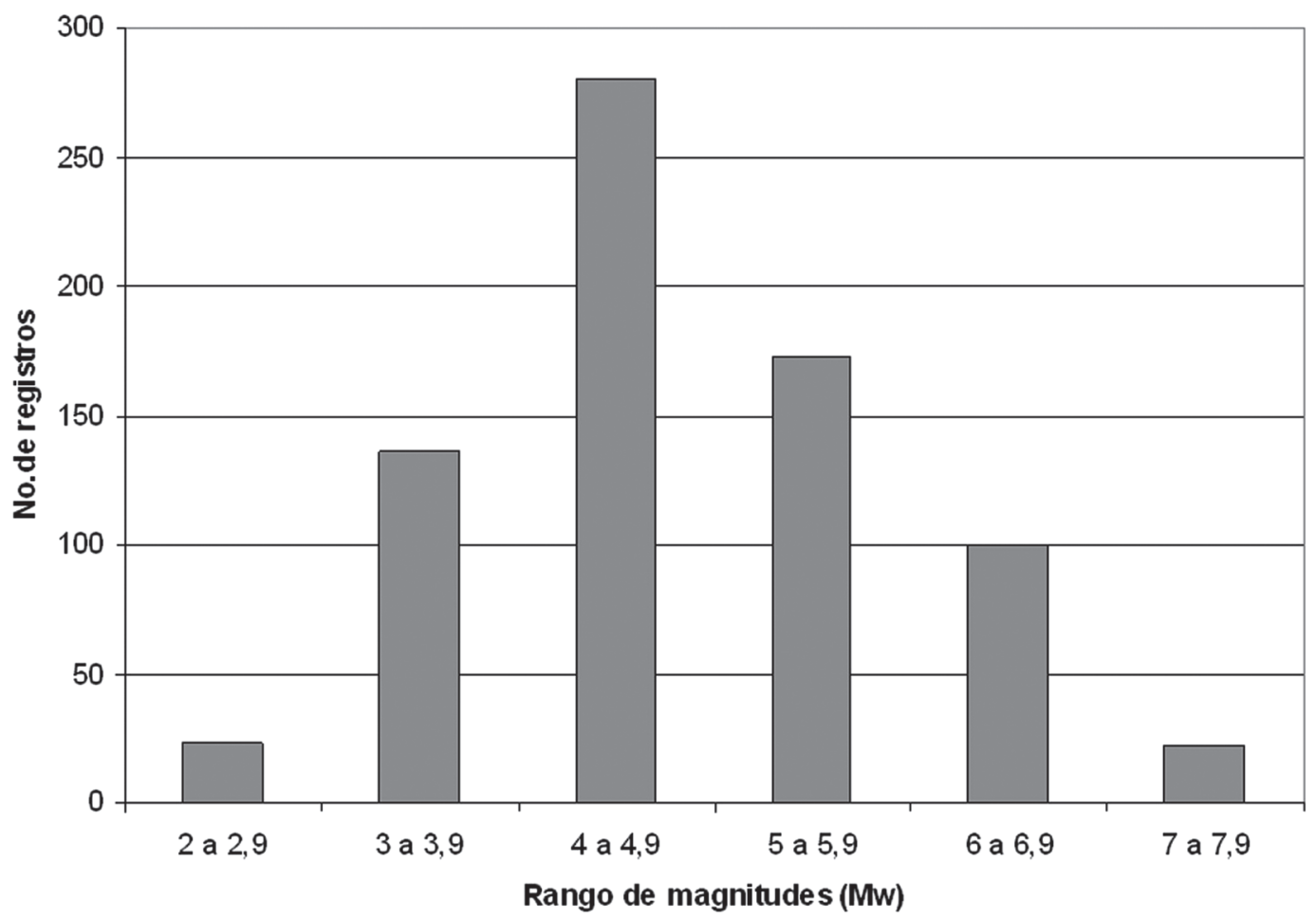

Fig. 4: Gráfico del número de registros respecto a rangos de magnitudes correspondientes a los registros seleccionados para este análisis. Predominan datos en el rango de Mw 4 a 5.

de los 100 gals. Por otro lado, son muy pocos los datos que se alejan notablemente de la línea de tendencia, de ahí la buena correlación observada.

La ecuación de mejor ajuste entre el PGA y la IA propuesta es entonces:

$$
I A=8 * 10^{-6} *(P G A)^{1,9956}
$$

La desviación estándar para este caso es: $\sigma=$ 0,0955 y el coeficiente de correlación $\mathrm{r}^{2}=0,9228$.

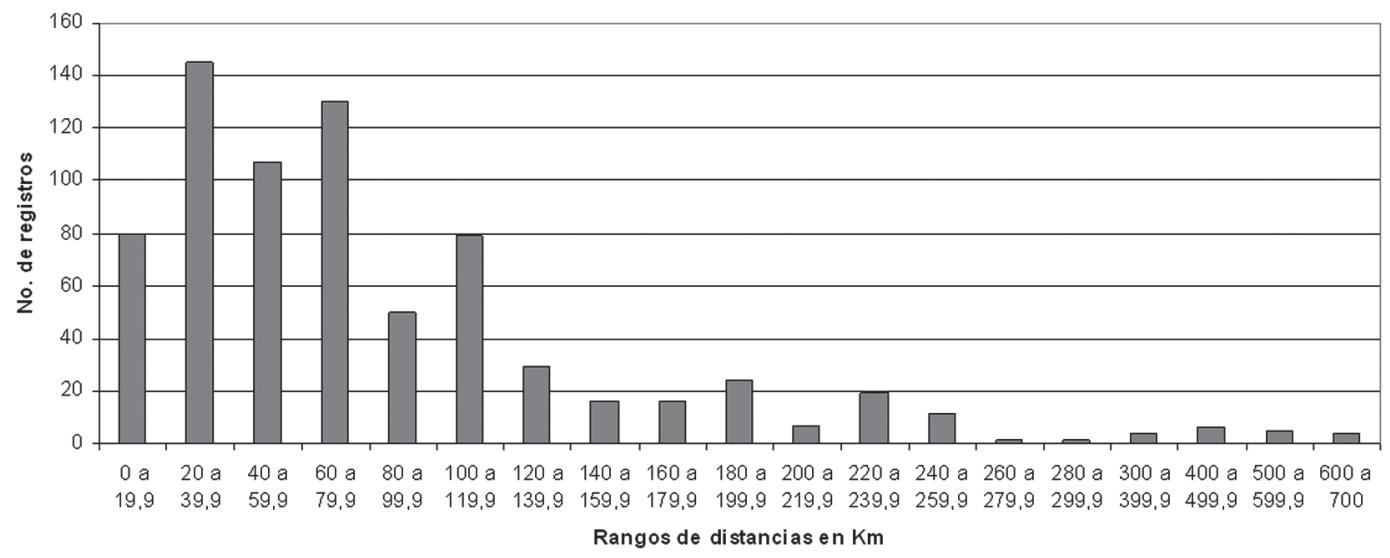

Fig. 5: Gráfico del número de registros respecto a rangos de distancia hipocentral para los datos seleccionados. Las distancias que predominan van de 20 a $80 \mathrm{~km}$. 


\section{Relaciones empíricas de atenuación para la Intensidad de Arias}

Las regresiones se llevaron a cabo por el método de mínimos cuadrados y en un solo paso, a partir de la ecuación 8 que es igual a la ec. 6 sin el término $\mathrm{c}_{\mathrm{p}}$ :

(8) $\ln Y=c_{0}+c_{m} M-c_{L D} \ln D-c_{D} D+c_{S} S$

Como se ha dicho, para el cálculo se contó con 734 registros y los coeficientes a determinar son entonces: $\mathrm{c}_{0}, \mathrm{c}_{\mathrm{m}}, \mathrm{c}_{\mathrm{D}}, \mathrm{c}_{\mathrm{LD}} \mathrm{y} \mathrm{c}_{\mathrm{s}}$, con Y como variable dependiente, en este caso igual a IA y con M, D y S como variables independientes. La correlación entre estos términos representa realmente un modelo de atenuación para IA.

La distribución de $\ln Y$ es muy cercana a la normal, excepto en el rango de IA entre 1,7E-5 $\mathrm{m} / \mathrm{s}$ y $4,5 \mathrm{E}-5 \mathrm{~m} / \mathrm{s}$, en el que se acumula una significativa cantidad de datos de IA que hacen que la tendencia de la distribución normal esté por debajo de estos valores. Esto representa una presencia de IA más bajas que las esperadas para el rango mencionado, que corresponde a distancias alrededor de los $100 \mathrm{~km}$, claramente observable en la figura 12 donde para distancias cercanas a los $100 \mathrm{~km}$ e IA de $1 \mathrm{E}-5 \mathrm{~m} / \mathrm{s}$, los puntos que corresponden a los valores observados son bastante inferiores a los que se obtienen por medio de las regresiones (curvas).

El término $\mathrm{D}$ que corresponde a la distancia hipocentral, fue ligeramente modificado de la siguiente forma:

$$
D=\sqrt{D_{h i p}^{2}+r^{2}}
$$

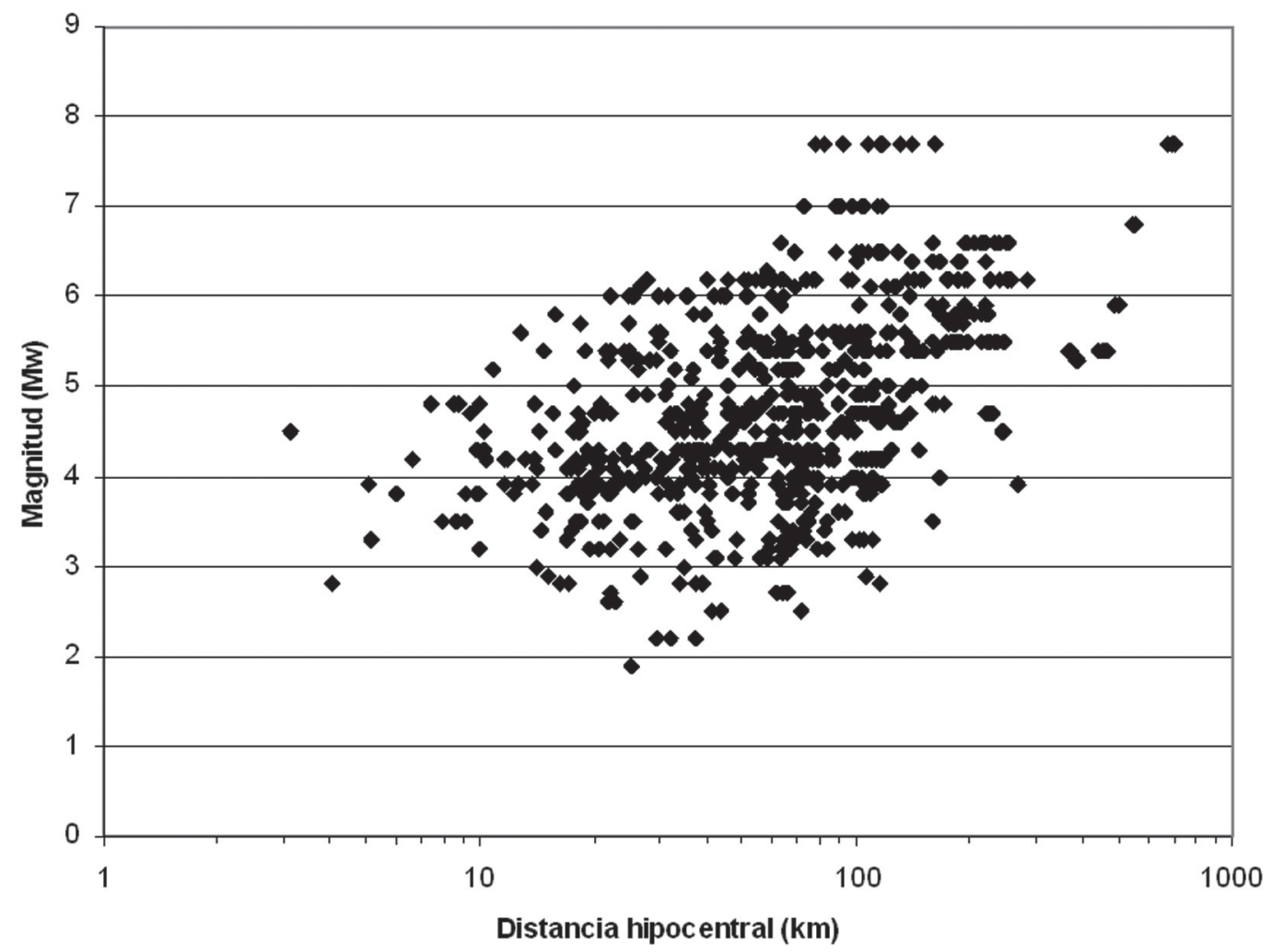

Fig. 6: Distribución de datos según su magnitud Mw respecto a la distancia hipocentral para el grupo de datos seleccionados para este estudio. 
a)

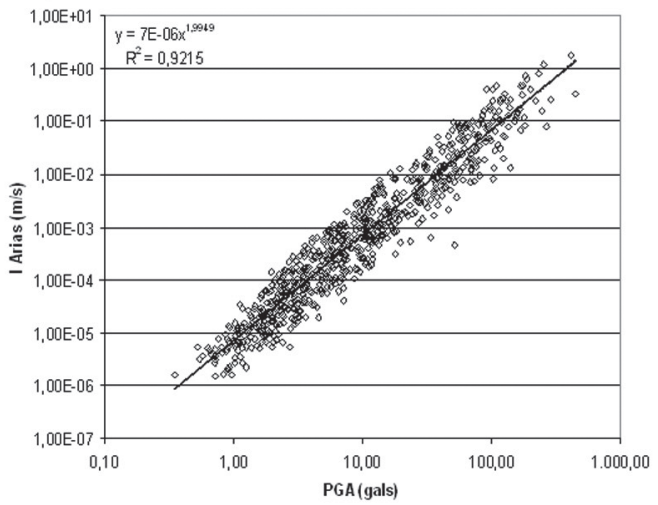

b)

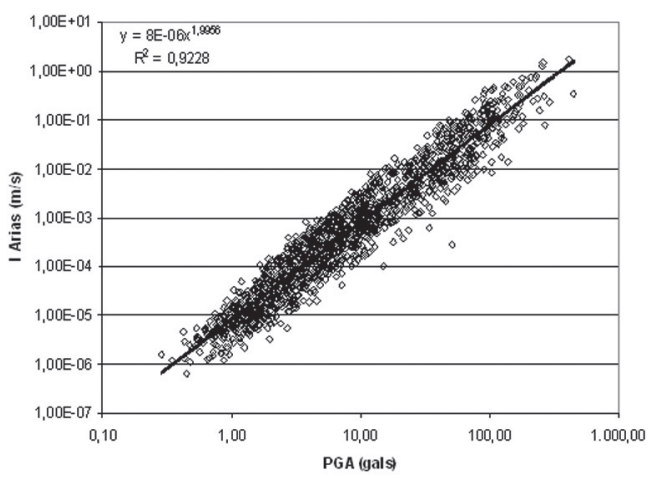

Fig. 7: Correlación entre PGA e IA para: .a) las IA máximas (un IA por registro) y b) ambas componentes horizontales por registro.

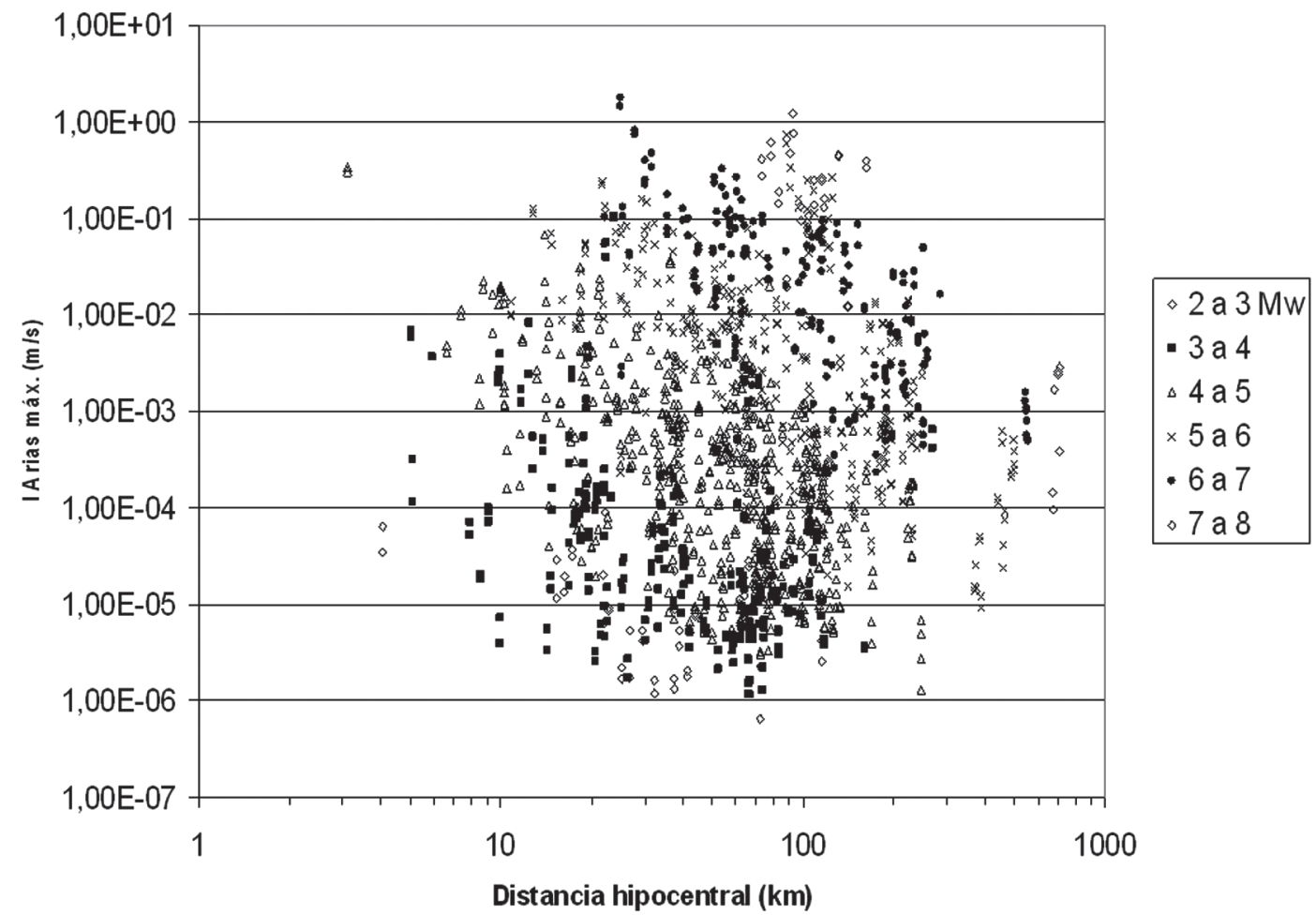

Fig. 8. Gráfico correspondiente a la IA máxima calculada para cada registro vs. la distancia hipocentral asociada, para todos los rangos de magnitudes considerados. 
Donde $r$ es una distancia ficticia, introducida para asegurar la estabilidad del modelo en el campo muy cercano, que corrige el término de las distancias cuando estas son muy cortas (sitios muy cercanos a la fuente). En vista de que hay muy pocos datos disponibles para distancias cortas (situación bastante común cuando se hace este tipo de análisis), esta distancia se adoptó igual a 6 km, tal y como lo propusieron Dahle et al., (1995) y Schmidt et. al., (1997), válida aún cuando se trabaja con distancias hipocentrales, ya que se logra definir el movimiento del suelo como constante en el campo muy cercano.

La Fig. 8 muestra la distribución de valores de IA vs. distancia hipocentral, a partir de los datos con los cuales se llevó a cabo la inversión. A pesar de parecer una nube de puntos, al observar este gráfico detalladamente se notan tendencias sobre la forma en que la IA se atenúa con la distancia para cada rango distinto de magnitudes. El caso que presenta mayor dispersión (menor claridad respecto a esta tendencia) es el rango de magnitudes entre 3 y 4 . Después de una revisión detallada de los datos (características del registro, magnitud asignada, cálculo de IA, características del instrumento), aún persiste esta amplia dispersión en algunos casos, sobre todo de IA altas para distancias intermedias, entre 10 y $40 \mathrm{~km}$ (Fig. 9a). Sin embargo, hay que recordar que la mayor cantidad

\section{a)}

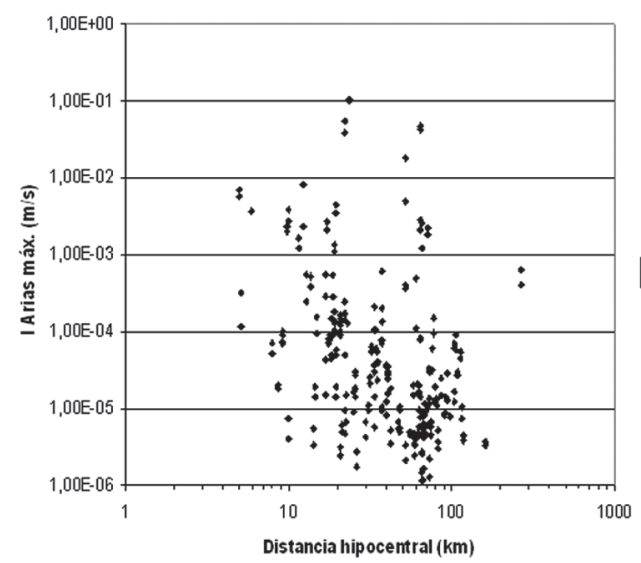

de datos disponible corresponde a magnitudes entre 4 y 5 , tal y como se mostró en la Fig. 4 y que sí presentan una clara tendencia a la atenuación con la distancia, como se muestra en la Fig. 9b.

Al realizar las regresiones se obtuvieron los resultados mostrados en el cuadro 2. Se consideraron varios casos para buscar el mayor coeficiente de correlación $\left(\mathrm{r}^{2}\right)$. De acuerdo con este parámetro, las correlaciones son bastante semejantes, disminuyendo un poco al separar los datos según las condiciones de suelo y por tipo de fuente.

El mejor resultado corresponde a la eliminación de los registros únicos o sea, al no considerar en el análisis los casos en que al ocurrir un sismo, solamente se obtiene un acelerograma en un único instrumento. Esta selección es una práctica usual debido a que se considera que la existencia de un solo registro acelerográfico no explica en forma adecuada el proceso de atenuación para un evento específico y no tiene un significado realista en el modelo general. Sin embargo, en vista de que en el presente caso el número de sismos que tienen esta condición es significativo y que por lo tanto, la cantidad de registros disminuiría considerablemente a la hora de hacer la inversión, se tomará como el caso más adecuado el correspondiente al primero del cuadro 2: utilizando la IA máxima de las dos componentes sin eliminar registros únicos.

\section{b)}

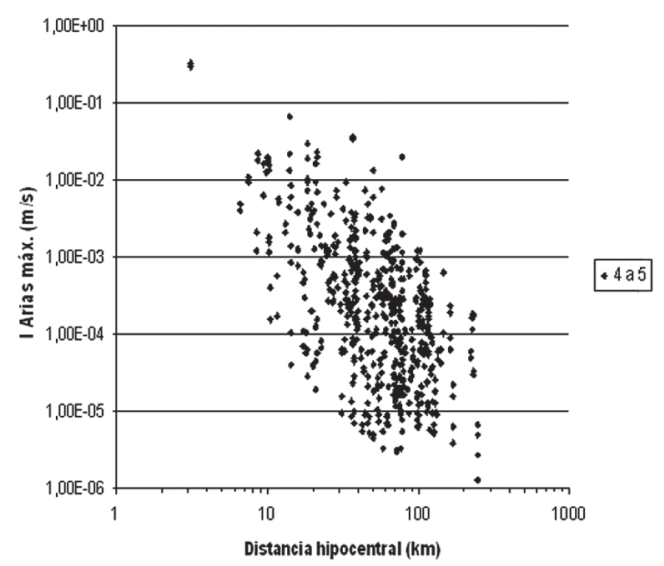

Fig. 9: Gráficos de IA máxima vs. distancia hipocentral para dos rangos de magnitudes distintas: a) entre 3 y 4 con una evidente dispersión donde se observan altos valores de IA para distancias intermedias y b) entre 4 y 5 donde se evidencia más claramente una tendencia de atenuación de los datos de IA. 


\section{Cuadro 2}

Casos considerados y coeficientes obtenidos para las regresiones correspondientes de IA respecto a la magnitud, distancia hipocentral y tipo de suelo

\begin{tabular}{cccccccc}
\hline Caso & $\mathrm{c} 0$ & $\mathrm{~cm}$ & $\mathrm{cLD}$ & $\mathrm{cD}$ & $\mathrm{cs}$ & $\mathrm{r} 2$ & $\begin{array}{c}\text { Error están- } \\
\text { dar }\end{array}$ \\
\hline $\begin{array}{c}\text { IA máximo de las } \\
\text { dos componentes }\end{array}$ & $-13,799$ & 2,685 & $-1,611$ & $-0,0034$ & 0,945 & 0,71 & 1,668 \\
$\begin{array}{c}\text { IA usando las dos } \\
\text { componentes }\end{array}$ & $-14,335$ & 2,612 & $-1,420$ & $-0,0042$ & 0,952 & 0,68 & 1,739 \\
$\begin{array}{c}\text { IA promedio } \\
\text { cuadrático (1) }\end{array}$ & $-13,846$ & 2,612 & $-1,443$ & $-0,0041$ & 0,945 & 0,68 & 1,733 \\
$\begin{array}{c}\text { IA promedio } \\
\text { aritmético (2) }\end{array}$ & $-14,253$ & 2,613 & $-1,434$ & $-0,0041$ & 0,947 & 0,68 & 1,730 \\
$\begin{array}{c}\text { Eliminando regis- } \\
\text { tros “únicos” (3) }\end{array}$ & $-13,287$ & 2,564 & $-1,514$ & $-0,0043$ & 0,784 & 0,72 & 1.590 \\
$\begin{array}{c}\text { Sin considerar tipo } \\
\text { de suelo (cs=0) }\end{array}$ & $-13,970$ & 2,665 & $-1,419$ & $-0,0041$ & ----- & 0,66 & 1,799 \\
$\begin{array}{c}\text { Para datos de roca } \\
\text { y firme solamente } \\
\text { Para datos de }\end{array}$ & $-13,461$ & 2,553 & $-1,545$ & $-0,0031$ & ----- & 0,62 & 1,810 \\
blando solamente & $-13,578$ & 2,666 & $-1,379$ & $-0,0050$ & ----- & 0,71 & 1,663 \\
\hline
\end{tabular}

(1) en vista de que se cuenta con dos componentes por registro, IA se calcula como ; (2) usando IA = (IAL+IAT)/2; (3) Hay varios casos en los que solamente existe un único registro de un sismo, por lo que es posible mejorar el ajuste eliminado estos registros.

No se obtienen mejores resultados si se trabaja con ambas componentes y se calculan los promedios cuadráticos y aritméticos correspondientes. Tampoco mejora la regresión si se separan los datos según la condición del suelo y se hacen inversiones independientes, o bien, al no considerar del todo el tipo de suelo donde fue obtenido cada registro. La separación por tipo de fuente tampoco mejora los resultados ya que se obtuvo un $r^{2}=0,599$, por lo que ni siquiera se incluye este caso en el cuadro 2.

Se obtuvieron las curvas para distintas magnitudes, comparándolas con todo el grupo de datos utilizado, en condición roca-suelo firme (Fig.10). Hay un efecto de suavizado en las líneas en el campo cercano, producto de introducir una distancia ficticia para predecir el movimiento a distancias cortas (menores que $6 \mathrm{~km}$ ), donde precisamente hay ausencia de datos. Se han trazado las líneas para la primera regresión obtenida: IA máxima, por las razones expuestas anteriormente.

Las Figs. 11a, b, c y d muestran las mismas curvas de la Fig. 10 pero separadas por rango de magnitudes. En la 11b se observa que el rango de
4,5 a 5,4 es el que contiene el mayor número de datos. Por otro lado, la figura $11 \mathrm{~d}$ muestra la buena aproximación de la atenuación anelástica que hace el modelo, para distancias superiores a los $100 \mathrm{~km}$. El mayor alejamiento de la línea de regresión obtenida se observa para magnitudes entre 3,5 a 4,4 (Fig. 11a) que se debe a una notable dispersión de los datos, como ya se había comentado.

En la figura 12 se presentan los resultados obtenidos considerando el tipo de suelo. A pesar de que los datos (diamantes blancos y negros para las condiciones de suelo blando y firme, respectivamente) no muestran una contundente diferencia que indique la presencia de amplificación debida a suelos blandos respecto a los firmes, las trazas resultantes sí muestran este efecto, ubicándose la de suelo blando por encima de la correspondiente a firme, como era de esperarse. El gráfico se hizo para magnitud igual a 5 .

Paralelamente, se llevó a cabo el cálculo de los residuos como la diferencia entre la IA observada (calculada a partir de todos los acelerogramas) y la IA estimada a partir de las regresiones. 


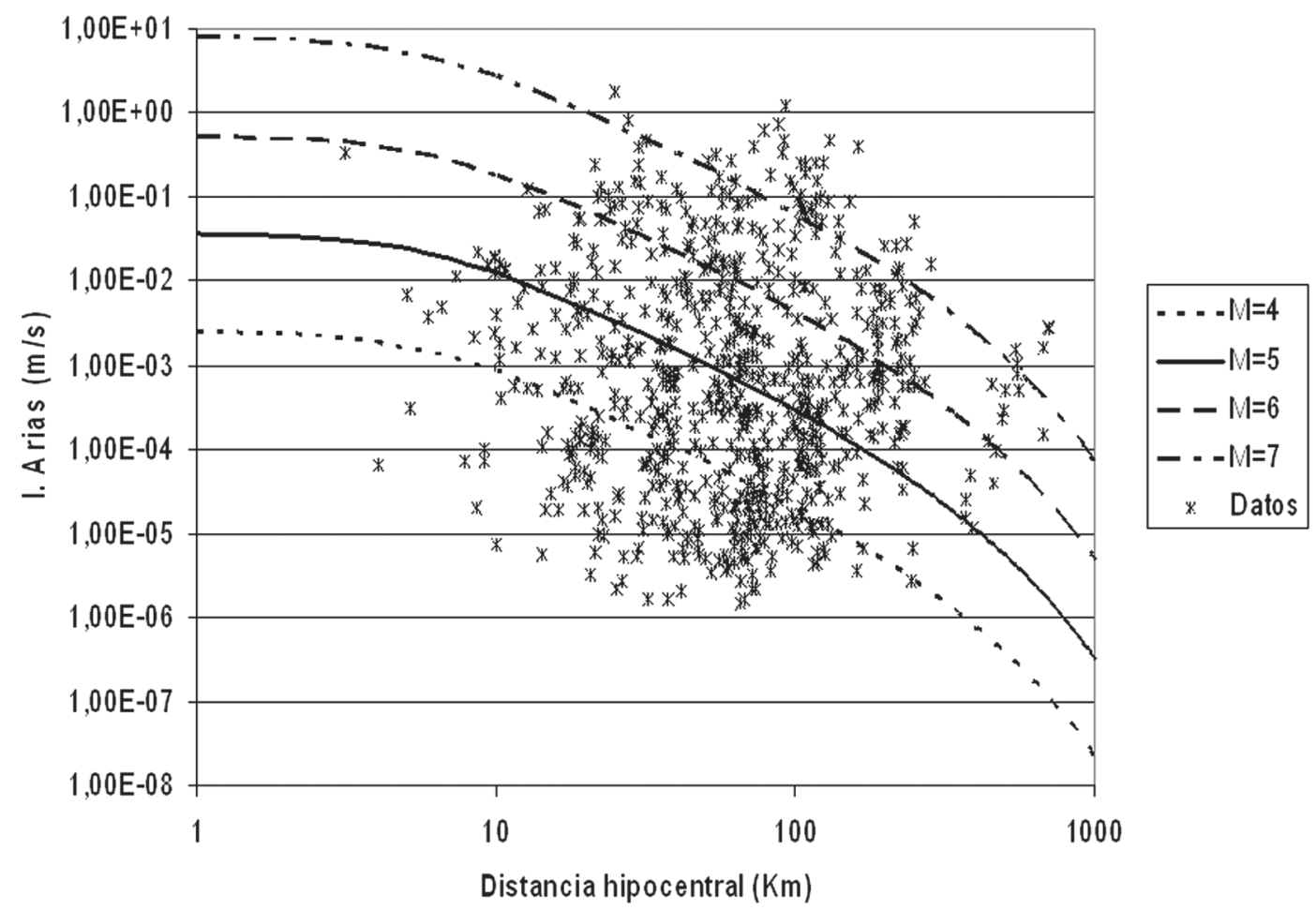

Fig. 10: Gráfico representando los datos correspondientes a IA máxima (datos reales) junto con las curvas de atenuación obtenidas por regresión para diferentes rangos de magnitud.

Los residuos se graficaron respecto a la distancia hipocentral (Fig. 13a) y a la magnitud (Fig. 13b). Se nota una tendencia hacia el valor cero que de hecho, luego de hacer los cálculos respectivos, representa la media geométrica. El caso que más se aleja de la media es el registro del terremoto de Alajuela (22/12/1990, MW $=6,0)$ registrado a una distancia hipocentral de $24,88 \mathrm{~km}$. Es a partir de este registro que se ha obtenido la mayor IA de todas las calculadas hasta la fecha con registros de Costa Rica $(\mathrm{IA}=1,74 \mathrm{~m} / \mathrm{s})$ y además, es el registro con mayor potencial de daño según distintos indicadores, como el propuesto por Park y Ang (Cruz et al., 1998).

Otros valores que muestran dispersión respecto a la media son los correspondientes a los registros del sismo de magnitud $\mathrm{Mw}=7,7$ (Terremoto de Limón, 22/04/1991), pero en estos casos la situación es a la inversa: los valores calculados son mayores que los observados; por lo tanto, las diferencias son negativas.
De acuerdo con la figura 13a, existe una sobreestimación general de valores por parte de las ecuaciones para distancias cercanas a los $100 \mathrm{~km}$. Esto puede estar asociado al hecho de que alrededor de esa distancia, los valores de IA observados decaen más fuertemente respecto a distancias menores, situación ya comentada. También, en la figura 13b es evidente la sobreestimación de IA para magnitudes entre 7 y 8 (residuos negativos), por lo que se puede concluir que las ecuaciones planteadas son más confiables para $\mathrm{Mw}$ inferior a 7, que son los casos donde los residuos son predominantemente cero.

Finalmente, se obtuvieron las comparaciones entre los resultados de este estudio y los obtenidos por Travasarou et al., (2003) y por Danciu \& Tselentis (2007), que representan las únicas relaciones semejantes obtenidas en la literatura disponible (Fig. 14). El origen de los datos utilizado por Travasarou et al., (2003) no se especifica en el documento consultado, pero parecen provenir de varias partes del mundo. Por su parte, los datos considerados por Danciu \& 
Tselentis (2007) corresponden a Grecia. Se comparan resultados medios (sin desviaciones estándar) para magnitudes de 5, 6 y 7 .

Se observa claramente una semejanza entre las tres curvas para magnitud 6 hasta los $60 \mathrm{~km}$ aproximadamente, a partir de lo cual los resultados para Costa Rica sufren una mayor atenuación respecto a las curvas obtenidas por los otros autores, situación que ya fue observada por Schmidt et al., (1997) para regresiones planteadas para PGA.

Para $\mathrm{Mw}=7$, este estudio sobreestima los valores de IA, en particular en el campo muy cercano (distancia hipocentral $<10 \mathrm{~km}$ ). Sin embargo, para $\mathrm{Mw}=5 \mathrm{y}$ hasta los $10 \mathrm{~km}$ la curva propuesta parece ser un promedio de las dos citadas como referencias, pero a partir de esta distancia se vuelve muy seme-
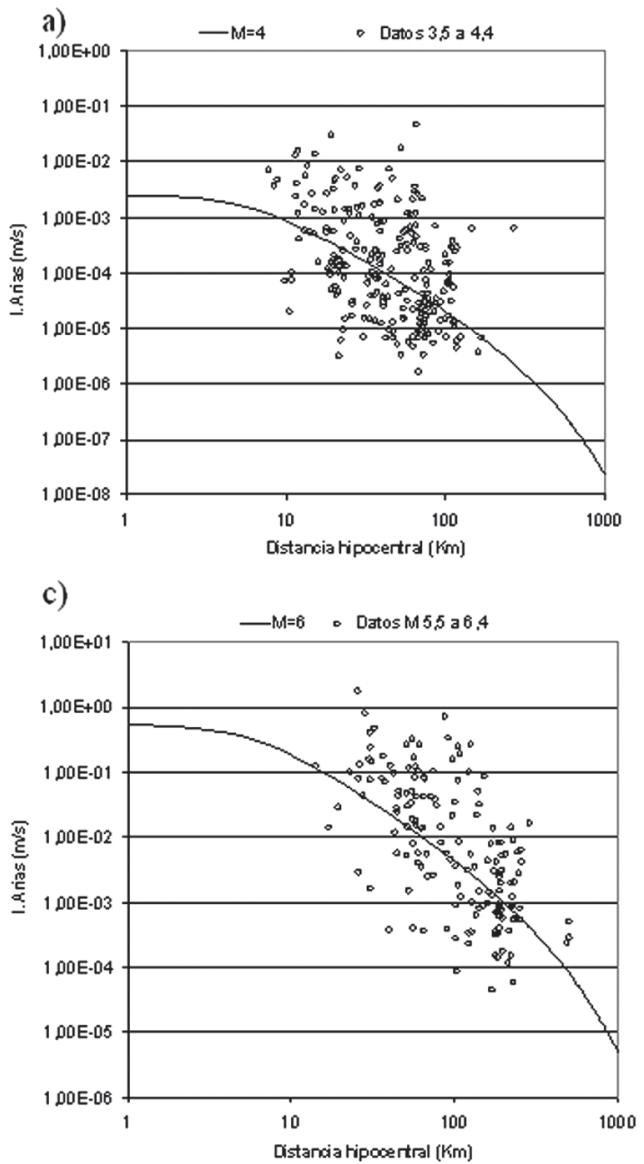

jante a la da Danciu \& Tselentis (2007), y ambas se ubican por debajo de la de Travasarou et al., (2003) quienes además proponen un modelo de atenuación menos fuerte a partir de los $100 \mathrm{~km}$.

\section{Correlaciones entre la IA y la IMM}

La última de las correlaciones que se propone en este estudio es la correspondiente a la de IA respecto a la IMM, a partir de los casos en los que se cuenta con ambos datos: el acelerograma registrado, con el que se calcula la IA y la IMM previamente asignada. Para este caso, se cuenta con 220 registros correspondientes a 81 eventos sísmicos sentidos, obtenidos entre 1987 y el 2004.
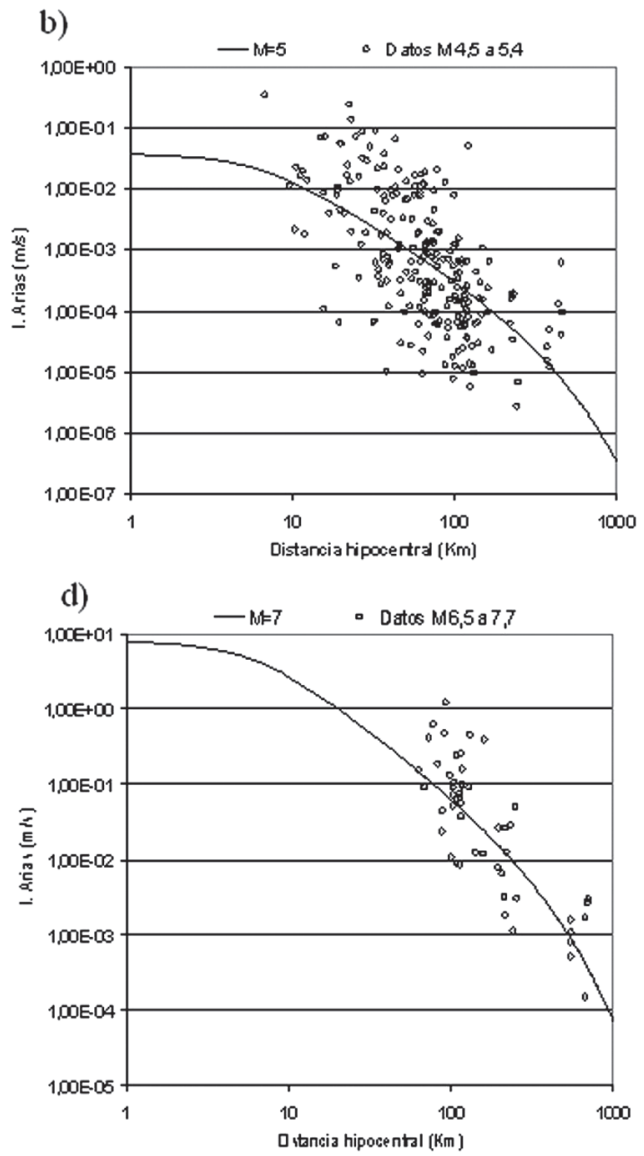

Fig.11: Gráficos de IA máximas vs. distancias hipocentrales separados por magnitud: a) $\mathrm{Mw}=4$,b) $\mathrm{Mw}=5$, c) $\mathrm{Mw}=6$ y d) $\mathrm{Mw}=7$. 


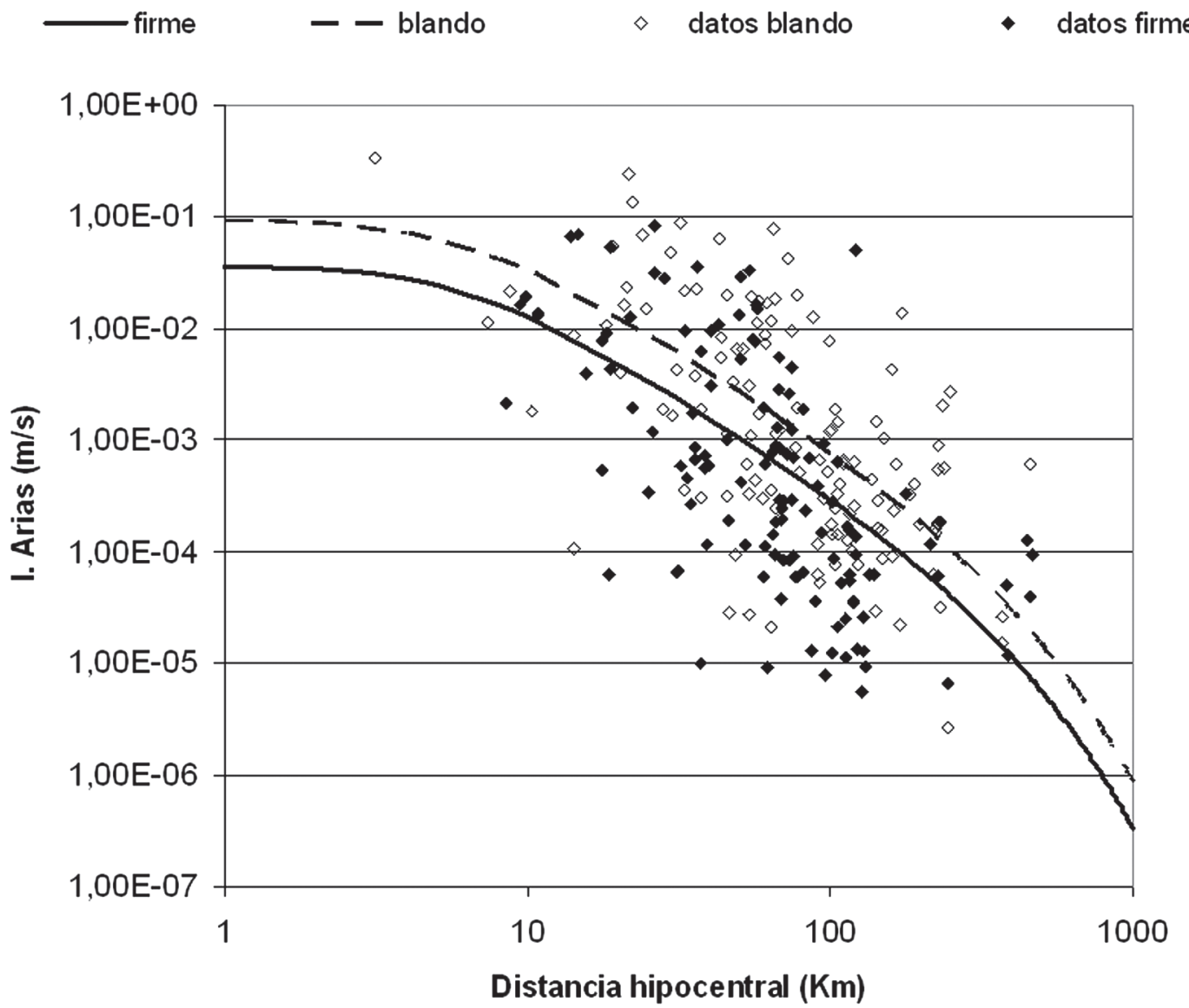

Fig 12: Comparación entre trazas correspondientes a las regresiones para suelo firme (traza continua) y para suelo blando (traza discontinua). Se muestran además los valores obtenidos a partir de datos reales para esas mismas condiciones de suelo.

a)

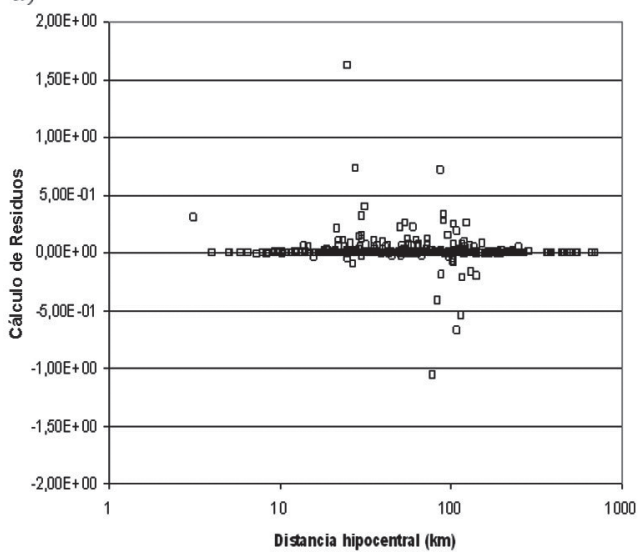

b)

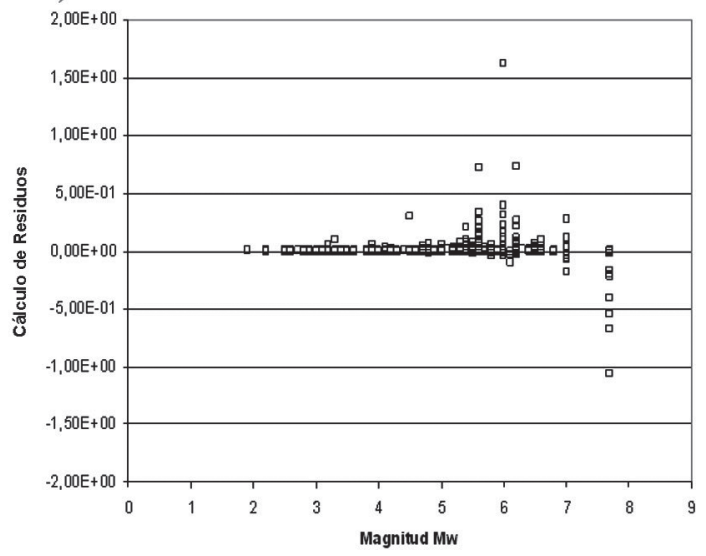

Fig. 13: Representación de los residuos de IA máxima, respecto a: a) distancia hipocentral y b) magnitud Mw. Los residuos en cada caso representan los valores calculados a partir de los registros menos los valores que predicen las ecuaciones obtenidas por regresión 


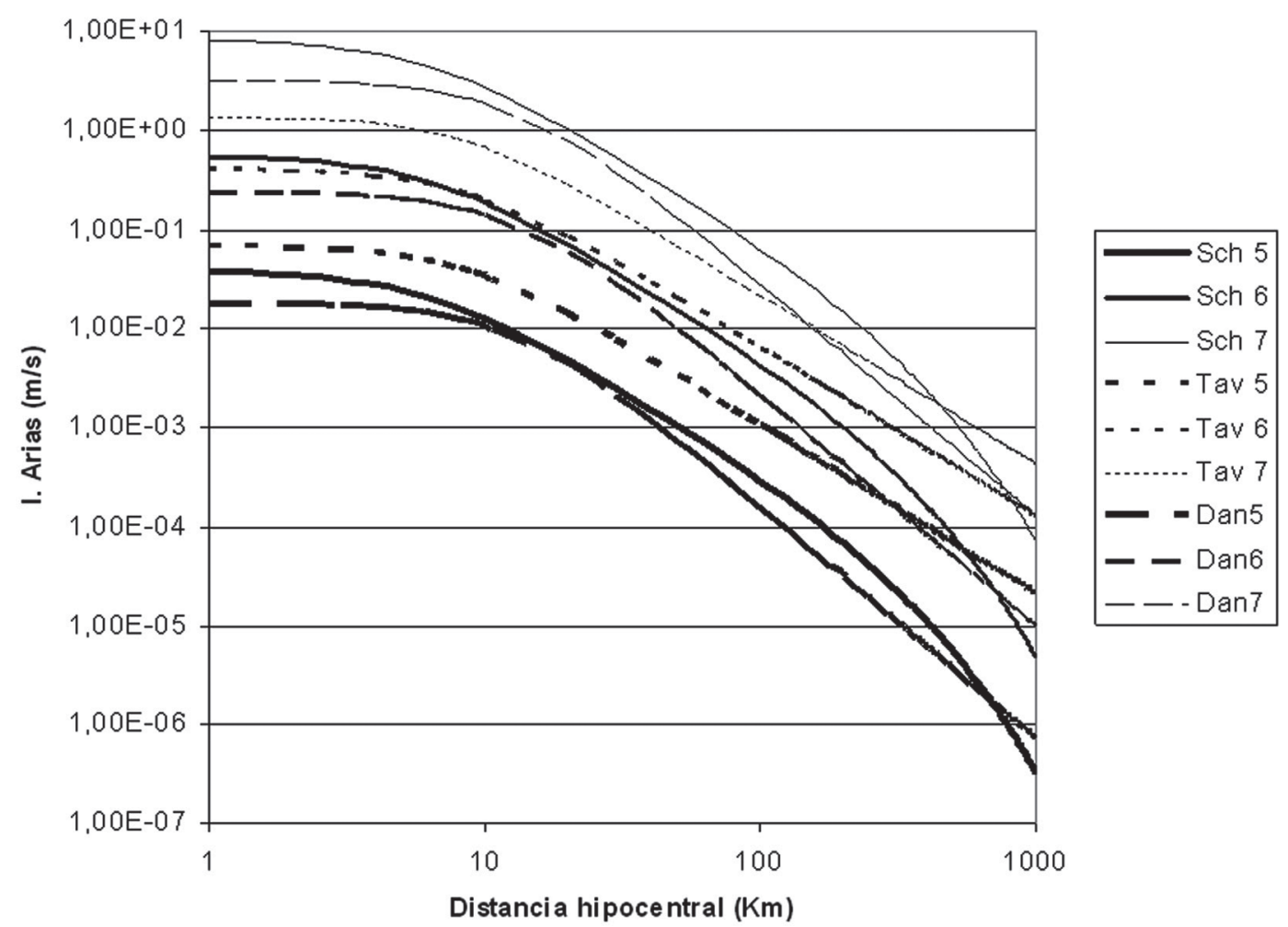

Fig. 14. Gráfico comparativo entre resultados de este estudio, trazas denominadas Sch 5, 6 y 7 con los obtenidos por Travasarou et al., (2002), (llamadas Tav 5,6 y 7) y por Danciu \& Tselentis (2007), (Dan 5, 6 y 7), para MW = 5, 6 y 7.

Todos los valores de IMM fueron tomados de la base de datos creada por Lepolt Linkimer quien analizó, uno a uno, los reportes de intensidad contenidos en mapas, artículos, periódicos, entrevistas, etc., referentes a estos 81 sismos (Linkimer, 2007).

La Fig. 15 muestra la distribución de datos de IA según la IMM. Se aprecia que es en el rango de intensidades de II a V donde se concentra la mayor cantidad de observaciones.

Para la estimación de la línea de mejor ajuste entre los datos, se llevaron a cabo varias pruebas. El primer caso es el mostrado en la Fig. 16, utilizando todos los valores de IA simultáneamente para cada IMM. El ajuste no logra ser muy significativo debido a dispersión de los datos con que se cuenta para cada grado de intensidad, por lo que se obtiene un bajo coeficiente de correlación, algo que se nota fácilmente en el gráfico al comparar visualmente los valores observados con la línea de regresión obtenida. Para este caso, la IA considerada por cada registro es la máxima, de las correspondientes a las dos componentes horizontales $\left(\mathrm{IA}_{\text {long }}\right.$ e $\left.\mathrm{IA}_{\text {tran }}\right)$.

Seguidamente, se llevó a cabo el ajuste considerando el promedio aritmético y luego, la media geométrica de la IA para cada valor de IMM. Los resultados son considerablemente mejores cuando se trabaja con la media geométrica y son los que se presentan en la Fig. 17. La Fig. 17a muestra los puntos de estas medias calculadas (diamantes) y la recta de mejor ajuste, cuyo coeficiente de correlación es de 0,9905. En la Fig. $17 \mathrm{~b}$ se presentan tanto los resultados de la ecuación como todo el grupo de datos y de ella puede observarse a simple vista, la coherencia de los resultados. Existe una gran dispersión entre los datos de IA para una misma IMM, situación que ha sido observada por otros autores (Boroschek \& Díaz, 2005) y es claro notar que esta dispersión aumenta conforme se llega a los valores más altos de intensidad. A pesar de esto, debe 


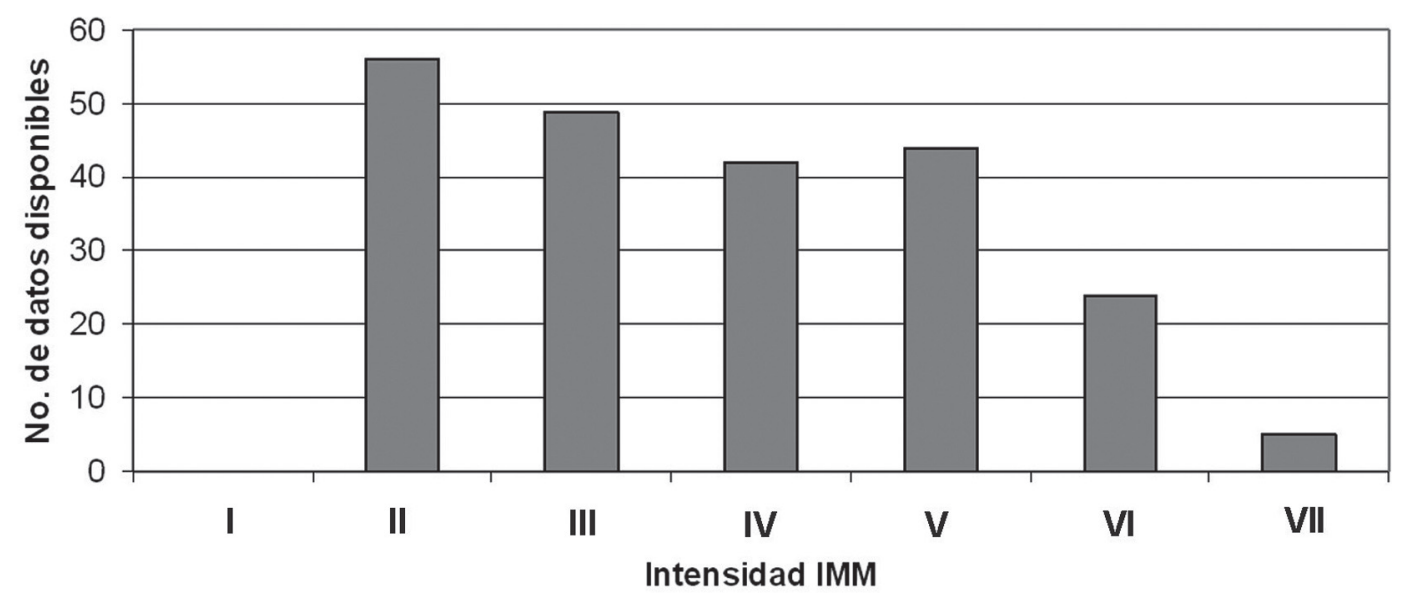

Fig. 15: Distribución de datos disponibles según la escala IMM. La mayoría se concentra entre IMM II y IV. Se observa que el rango de datos disponible para la IMM va de II a VII.

indicarse que la escala para IA es logarítmica, por lo que el rango de variación de datos disponibles de IA para IMM = VII es el máximo de todos $(0,139 \mathrm{~m} / \mathrm{s}$ a $1,74 \mathrm{~m} / \mathrm{s})$, mientras que el correspondiente a IMM = II es el menor (3,34x 10-6 $\mathrm{m} / \mathrm{s}$ a $1,54 \times 10-3 \mathrm{~m} / \mathrm{s})$.

En vista de que los cálculos anteriores se hicieron a partir de las IA máximas por registro, se volvió a hacer el análisis pero considerando el vector cuadrático para IA, o sea, $\mathrm{IA}_{\text {vector }}=\sqrt{I A^{2} \log _{3}+I A_{m a n}^{2}}$, debido a que hay investigadores que prefieren tratar de esta manera los datos cuando cuentan con las estimaciones de IA derivadas de las dos componentes horizontales. Los resultados obtenidos, así como el coeficiente $\mathrm{r}^{2}$, se presentan en el cuadro 3.

Con base en los coeficientes de correlación, se desecha la ecuación obtenida utilizando todo el grupo de datos y se consideran válidas las correspondientes a la media geométrica para $\mathrm{IA}_{\text {máx }}$ y para $\mathrm{IA}_{\text {vector }}$, debido a que tienen un $\mathrm{r}^{2}$ muy se- mejante. El uso de una u otra ecuación producirá, tal y como lo muestra la figura 18, un valor ligeramente mayor de IA para una misma IMM, en caso de que se utilice la ecuación correspondiente a la $\mathrm{IA}_{\text {vector }}$ debido precisamente a que este valor será siempre mayor que IA máx . La elección de una u otra correlación queda a discreción del investigador. En este caso no se llevó a cabo un cálculo de residuos debido a la evidente correlación que muestran los datos con la regresión obtenida al utilizar la media geométrica para IA.

En las Figs. 18 a, b, c y d se muestran comparaciones entre la correlación obtenida con todo el grupo de datos, respecto a las que se han estimado considerando subgrupos: primero separándolos según el origen (fallamiento local y subducción) y luego por el tipo de suelo (firme o blando).

Al considerar el tipo de fuente (Figs. 18a y b) los resultados se asemejan mucho a la regresión obtenida utilizando todo el conjunto de datos sin distinción, subestimándose levemente los valores

Cuadro 3

Ecuaciones obtenidas para correlacionar IMM con IA.

\begin{tabular}{ccc}
\hline Caso & Ecuación & Coeficiente r2 \\
\hline Media geométrica para IAmáx & $\mathrm{IMM}=0,5719 * \ln (\mathrm{IA})+7,1952$ & 0,9905 \\
Media geométrica para IAvector & $\mathrm{IMM}=0,5667 * \ln (\mathrm{IA})+6,9362$ & 0,9901 \\
Todo el grupo de datos & $\mathrm{IMM}=0,4148 * \ln (\mathrm{IA})+6,2279$ & 0,7679 \\
\hline
\end{tabular}




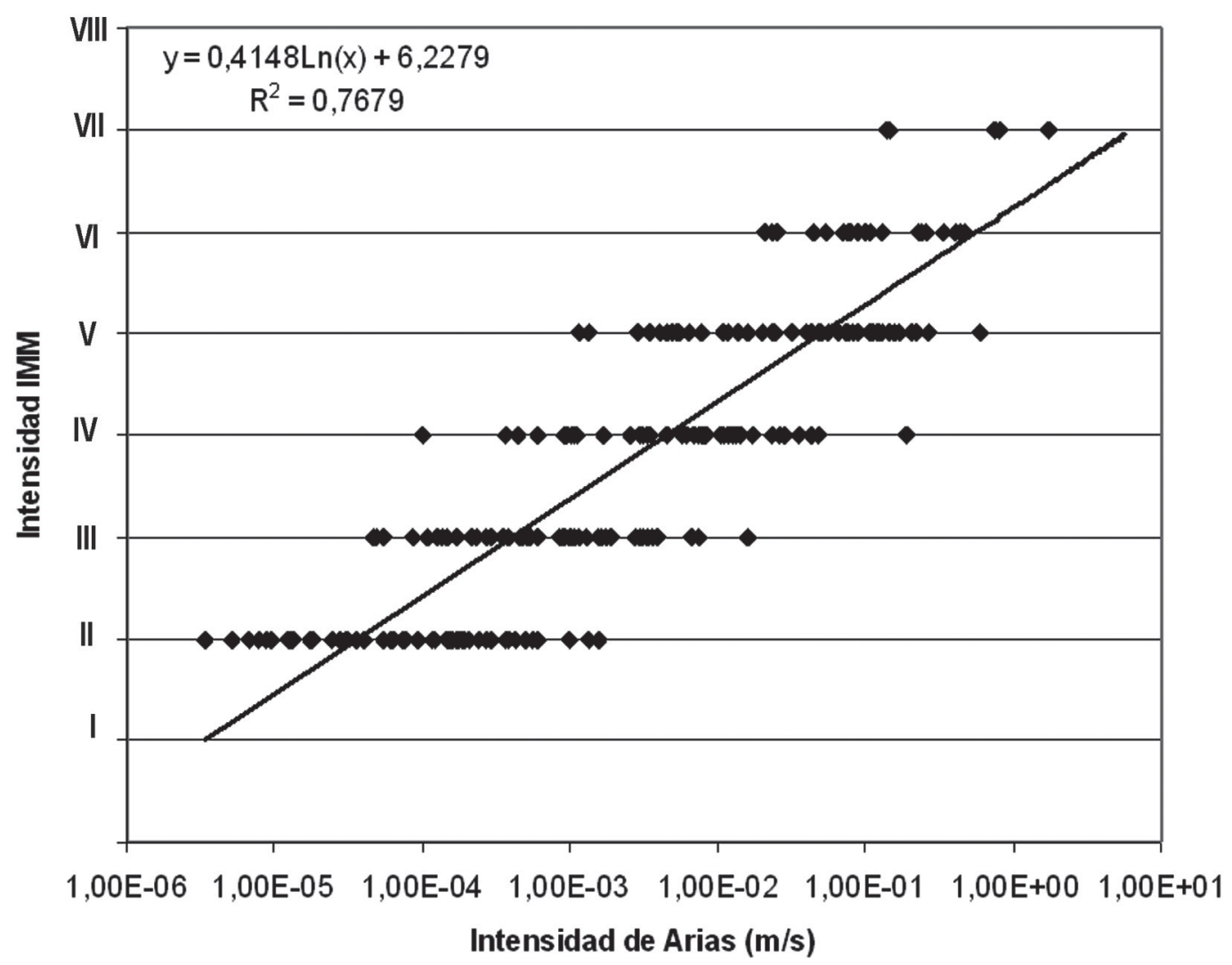

Fig.16: Correlación entre IMM e IA máxima calculada para todo el grupo de datos, sin cálculos de promedios de IA para cada IMM.

a)

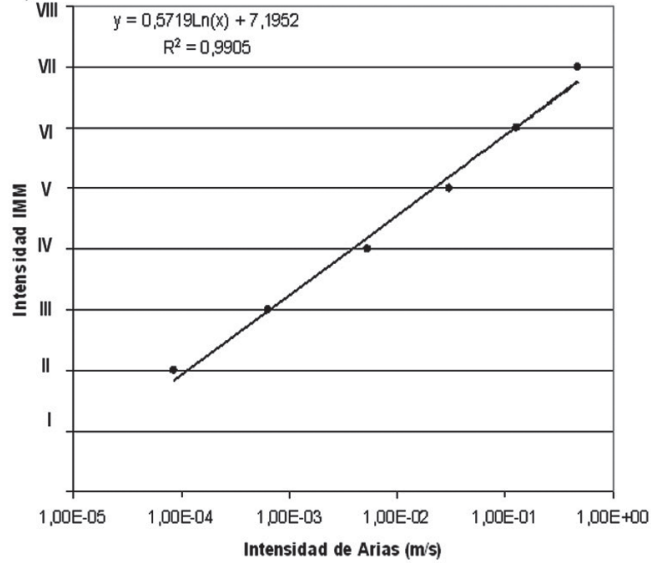

b)

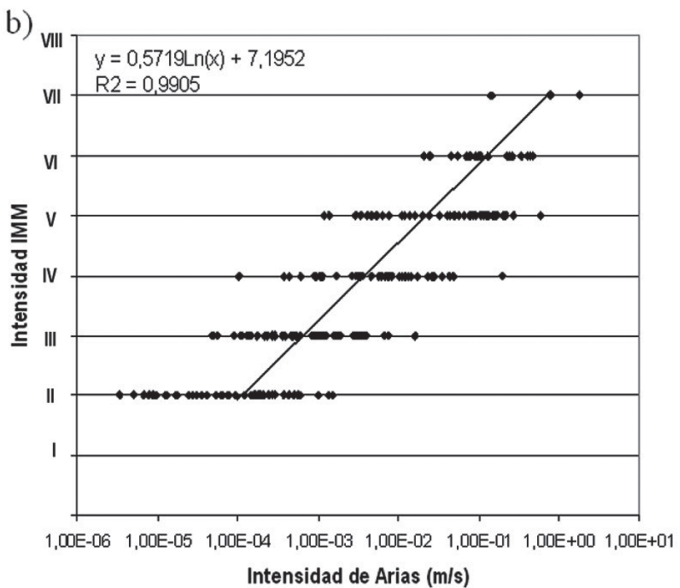

Fig. 17: Gráficos correspondientes a los mejores resultados obtenidos: a) línea de mejor ajuste utilizando valores de media geométrica de IA para cada IMM, b) ecuación graficada respecto a la totalidad de los datos considerados. 

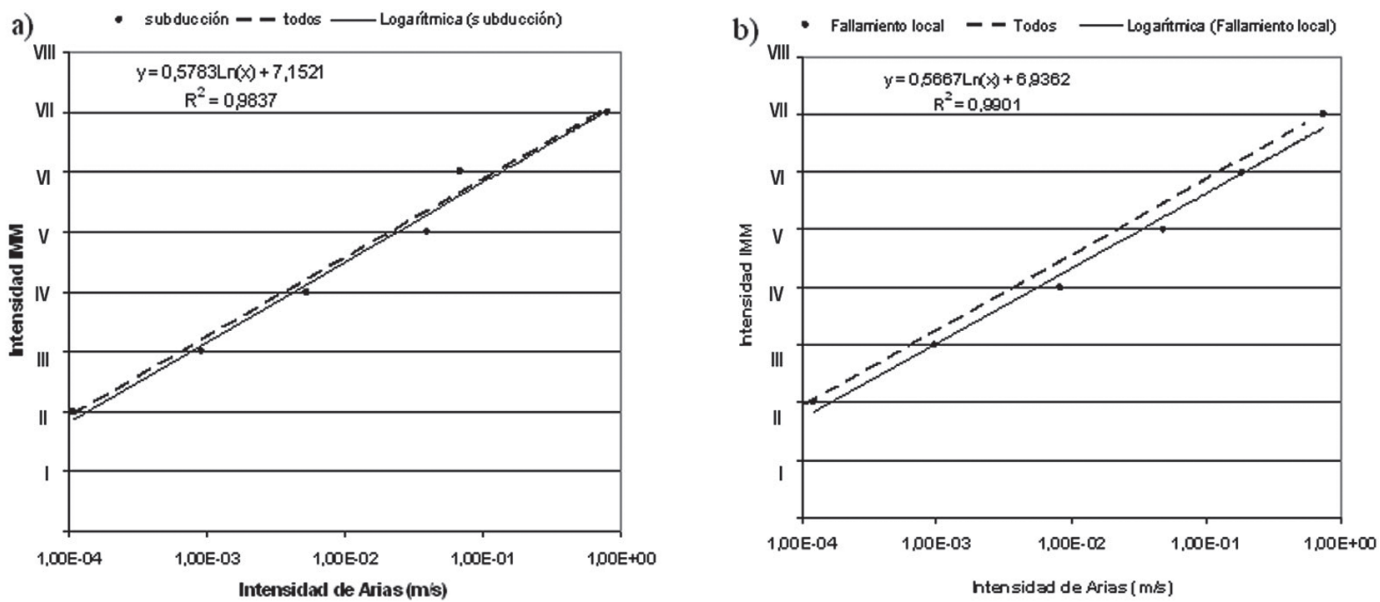

c) Suclo fime - - Todos - Logeritunica(Suelo fime)
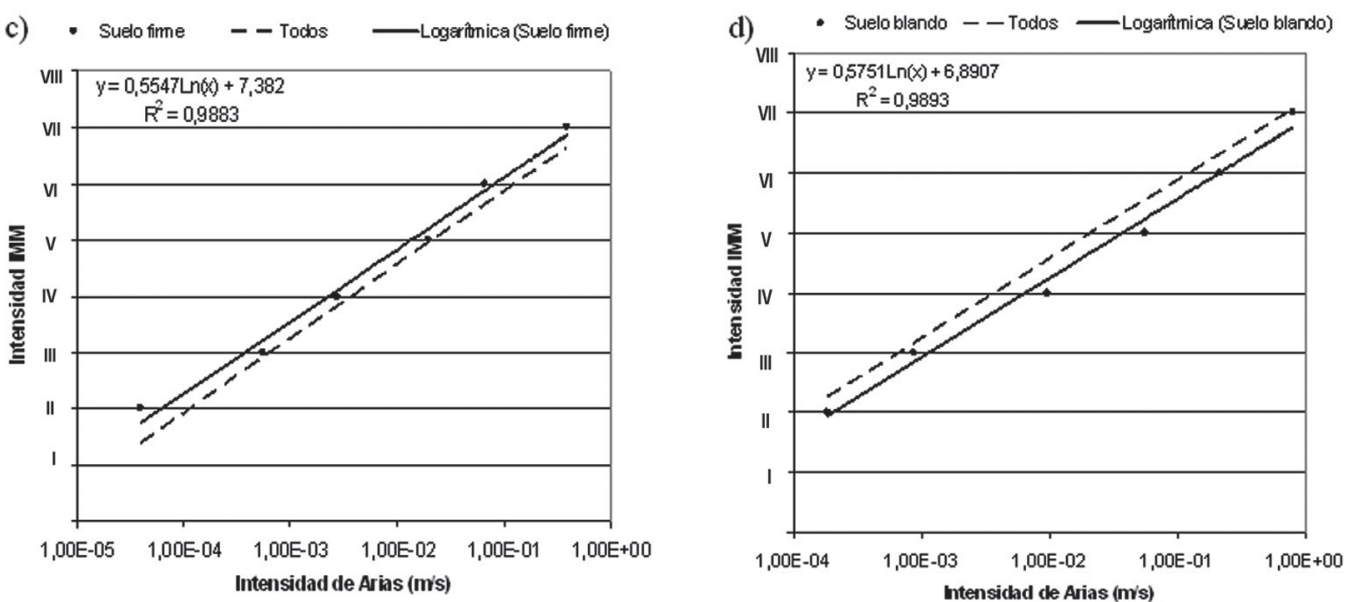

Fig. 18: Comparación de las regresiones obtenidas para la totalidad de los datos respecto a subgrupos escogidos: a) subducción, b) fallamiento local, c) suelo firme y c) suelo blando.

con el uso de la ecuación obtenida a partir de los datos correspondientes a fallamiento local respecto al grupo total de datos.

Al separar los datos según las condiciones del suelo existentes en la estación, se observa que para suelo firme, los valores de IMM son sobreestimados respecto a la regresión obtenida con el grupo completo de datos, debido a que las pendientes de ambas rectas son aproximadamente iguales pero la correspondiente a suelo firme siempre queda por encima, por ser mayor la ordenada en el origen (Fig. 18c). Esta situación se invierte cuando se analizan los datos referentes a suelo blando (Fig. 18d), en la que se evidencia que la regresión obtenida a partir de este grupo de datos subestima la ecuación propuesta a partir del grupo completo, siendo de nuevo ambas rectas bastante paralelas. En todos estos casos se trabajó con la media geométrica de IA para cada valor de IMM y con la IA máxima por componente.

La Fig. 19 muestra comparaciones entre las regresiones obtenidas en este estudio y seleccionadas del cuadro 3 , con las propuestas por dos estudios previos: Cabañas et al., (1997) a partir de datos registrados en Italia y Boroschek \& Díaz (2005), con base en datos chilenos.

En términos generales, se observan tendencias muy semejantes entre la propuesta en esta investigación referente a IA máxima con respecto a la de Cabañas et al., (1997), a pesar de que 


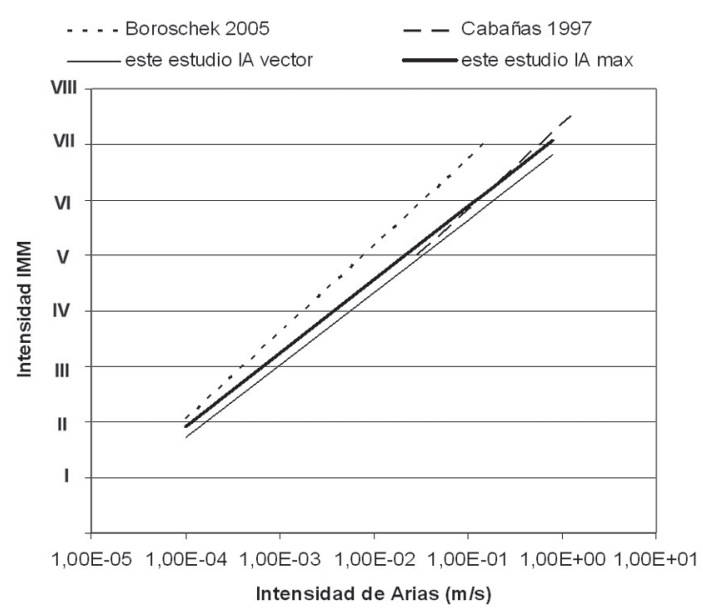

Fig. 19: Gráfico comparativo de distintas correlaciones entre IMM e IA, según el autor de este estudio, el de Cabañas et al., (1997) y el de Boroschek \& Díaz (2005).

esta última es estimada para un rango pequeño de intensidades entre V y VII. La generada en este estudio tiene una pendiente ligeramente menor, pero para el rango graficado de V a VII ambas ecuaciones dan resultados muy similares.

Al comparar los resultados de la presente investigación con los propuestos por Boroschek \& Díaz (2005), para IMM = II los resultados son muy semejantes, pero conforme aumenta la IMM, los valores de IA que estima Boroschek \& Díaz (2005) son menores que los obtenidos en este estudio, así como por los de Cabañas et al., (1997) debido a una menor pendiente según los datos de Chile. Esto significa por ejemplo, que para un cambio relativo de un grado de IMM a otro, las ecuaciones propuestas aquí estiman $\triangle I A$ mayores que las chilenas. Sin embargo, los resultados de este estudio parecen satisfactorios (con coeficientes de correlación altos) y claramente del mismo orden que otros propuestos, aún cuando se están comparando con ecuaciones obtenidas a partir de datos de otras partes del mundo, en condiciones geológicas y tectónicas distintas.

\section{CONCLUSIONES}

A lo largo de esta investigación, se trabajó con el parámetro denominado Intensidad de Arias (IA), ya que es considerado como un indicador preciso de la fuerza con que un sismo actúa en un sitio específico, estimado a partir de un registro acelerográfico dado.

El banco de datos inicial consta de 1018 registros acelerográficos de campo libre obtenidos entre 1983 y el 2006 (413 eventos con Mw entre $2,2$ y 7,7$)$. Luego de definir una serie de criterios de selección, el grupo de datos se redujo a 734 registros (320 sismos sentidos). La correlación obtenida entre la IMM con la IA se trabajó a partir de 220 registros correspondientes a 81 eventos sísmicos registrados entre 1987 y el 2004, con IMM asignadas entre II y VII según Linkimer (2007).

Los distintos valores de IA calculados para cada acelerograma se correlacionaron con otros parámetros del sismo, de las condiciones geológicas de la estación y de valores de intensidad en la escala Mercalli Modificada asociados con el sitio donde fue registrado. Se obtuvieron los siguientes resultados que han sido seleccionados como los más confiables de acuerdo con los parámetros estadísticos:

- Correlación entre IA y PGA, utilizando ambas componentes para PGA:

$\mathrm{IA}=8 \mathrm{E}-6 *(\mathrm{PGA})^{1,9956}, \mathrm{r}^{2}=0,923(\mathrm{IA}$ en $\mathrm{m} / \mathrm{s}$ y PGA en gals)

Esta ecuación permite calcular la IA a partir del valor de PGA proveniente de un acelerograma, sin necesidad de contar con todo el registro de aceleraciones en el tiempo.

- Correlación entre IA como variable dependiente $\mathrm{y}_{\mathrm{w}}$, D y $\mathrm{S}$ como variables independientes, utilizando IA como la máxima de las dos componentes horizontales:

$\operatorname{Ln}(\mathrm{IA})=-13,799+2,685^{*} \mathrm{Mw}-1,611 * \operatorname{Ln}(\mathrm{D})-$ $0,0034 * \mathrm{D}+0,945 * \mathrm{~S}, \mathrm{r}^{2}=0,71(\mathrm{IA}$ en $\mathrm{m} / \mathrm{s}$ y D en km)

Esta ecuación permite estimar la IA máxima en un sitio específico, a partir de conocer los siguientes parámetros: magnitud de momento (Mw), distancia hipocentral corregida $\left(D-\sqrt{D_{k p p}^{2}+6^{2}}\right)$ y el tipo de suelo $(S)$, siendo $S=0$ para condición de suelo firme y $\mathrm{S}=1$ para blando. De acuerdo con el análisis de residuos llevado a cabo, se obtuvo un valor medio de cero para $\mathrm{Mw}$ entre 2 y 7 , por lo que se recomienda usar la relación propuesta para magnitudes.inferiores a 7.

- Correlación entre IA e IMM, a partir de la media geométrica de IA máxima, para cada grado de IMM: 
$\mathrm{IMM}=0,5719^{*} \operatorname{Ln}(\mathrm{IA})+7,1952, \mathrm{r}^{2}=0,9905$ (IA en $\mathrm{m} / \mathrm{s}$, IMM es adimensional)

Permite calcular la IMM a partir del cálculo de IA máxima (máximo entre $\mathrm{IA}_{\text {long }}$ e $\mathrm{IA}_{\text {trans }}$ ) que es obtenida con base en un registro acelerográfico.

De los resultados obtenidos, se puede comentar lo siguiente:

A pesar de no encontrarse en la literatura resultados comparables respecto a la correlación obtenida entre IA y PGA, la ecuación muestra una gran estabilidad de acuerdo con el alto coeficiente de correlación y con la poca dispersión de los datos que se observa en los gráficos de las figuras $7 \mathrm{a}$ y $7 \mathrm{~b}$.

La regresión planteada entre IA, Mw, D y S se asemeja bastante a las propuestas por Travasarou et al., (2003) usando datos de distintas partes del mundo y por Danciu \& Tselentis (2007) con datos procedentes de Grecia, principalmente para $\mathrm{Mw}=6$ y distancias D entre 0 y $100 \mathrm{~km}$. Sin embargo, el presente estudio sobreestima los valores de IA para ese mismo rango de distancias para $\mathrm{Mw}=7 \mathrm{y}$ las subestima para $\mathrm{Mw}=5$ respecto a las que propone Travasarou et. al., (2003). En los tres casos comparados (Mw de 5,6 y 7), las ecuaciones aquí propuestas muestran una atenuación más fuerte que las otras dos a partir de los $100 \mathrm{~km}$.

- Al comparar las correlaciones obtenidas entre IA e IMM, se observan tendencias muy semejantes, en especial con la estimada a partir de datos de Italia que tiene validez en el rango de $\mathrm{V}$ a VII. Si se compara la ecuación propuesta a partir de datos de Chile con la que aquí se propone, ambas parten de una misma IA para IMM = II pero la de este estudio tiene una menor pendiente, lo que significa que para un mismo valor de intensidad entre III a VII, las propuestas para Costa Rica estiman IA mayores respecto a las chilenas, lo que puede reflejar el hecho de que los datos provienen de regiones con características distintas. Por otro lado, se observa una gran similitud con los resultados obtenidos por Cabañas et al., (1997) en las que, a pesar de provenir de marcos tectónicos distintos, se evidencian semejanzas. Por lo tanto, no se puede establecer como conclusión que las diferencias de los entornos traigan siempre como consecuencia resultados distintos.

\section{AGRADECIMIENTOS}

Este artículo fue escrito dentro del programa de doctorado en la Universidad Politécnica de Cataluña (UPC), con una beca otorgada por la Universidad de Costa Rica y complementos de beca por parte de la UPC, el MICIT y el CONICIT de Costa Rica. Un agradecimiento al Instituto Geológico de Cataluña por las facilidades para llevar a cabo esta investigación, en especial a los doctores Xavier Goula, Sara Figueras y Teresa Susagna, por sus valiosos comentarios. También al M.Sc. Lepolt Linkimer por facilitarme la base de datos referente a la asignación de intensidades para sismos de Costa Rica que él elaboró. A los revisores por sus útiles sugerencias. Asimismo, al personal del Laboratorio de Ingeniería Sísmica, quienes en forma incondicional estuvieron siempre atentos a enviarme la información que les haya solicitado, así como en la edición final de las figuras.

\section{REFERENCIAS}

ARIAS, A., 1970: A measure of earthquake intensity. - R.J. Hansen, ed. Seismic Design for Nuclear Power Plants, MIT Press. Cambridge, Massachusetts: 438-483.

BOORE, D. \& JOYNER, W., 1982: The empirical prediction of strong motion. - Bull. Seism. Soc. Am. 72(6): 843-860.

BOROSCHEK,R\&DÍAZ,A.,2005:Determinación experimental de la intensidad de Mercalli modificada para Chile. - Congreso chileno de Sismología e Ingeniería Antisísmica, IX jornadas. Concepción, Chile: 1-14. 
CABAÑAS, L., BENITO, B. \& HERRIAZ, M., 1997: An Approach to the measurement of the potential structural damage of earthquake ground motions. - Earth. Eng. Struct. Dynam. 26: 79-92.

\section{COLEGIO FEDERADO DE INGENIEROS} Y DE ARQUITECTOS DE COSTA RICA (CFIA), 2003: Código Sísmico de Costa Rica 2002 [3 $3^{\text {a }}$ ed.]. - 288 págs. Ed. Tecnológica de Costa Rica, Cartago.

CRUZ, M., INFANTE, E. \& SCHMIDT, V., 1998: Evaluación del Potencial de Daño de Sismos Recientes en Costa Rica. - Rev. Ing. 8 (1 y 2): 9-16.

DAHLE, A., CLIMENT, A., TAYLOR, W. BUNGUM, H., SANTOS, P, CIUDAD REAL, M., LINDHOLM, C., STRAUCH, F. \& SEGURA, F., 1995: New spectral strong motion attenuation models for Central America. - Proceedings of the 5th Int. Conf. Seism. Zon., Nice, France. 2: 1005-1012.

DANCIU, L \& TSELENTIS, G., 2007: Engineering ground-motion parameters attenuation relationships for Greece. - Bull. Seism. Soc. Am. 97 (1B): 162-183.
LINKIMER, L., 2007: Relationship between peak ground acceleration and Modified Mercalli intensity in Costa Rica. - Rev.Geol. Am. Cen. XXX: págs...

ROJAS, W. BUNGUM, H. \& LINDHOLM, C., 1993a: A catalog of historical and recent earthquakes in Central America. - 78 págs. 2-7. NORSAR, Norway [Tech. Report].

SAUTER, F., 1989: Introducción a la Sismología. -271 págs. Ed. Tecnológica de Costa Rica, Cartago.

SCHMIDT, V., DAHLE, A. \& BUNGUM, H., 1997: Costa Rican spectral strong motion attenuation. - 45 págs. NORSAR, Norway [Tech. Report].

TRAVASAROU, T., BRAY, J. \& ABRAHAMSON, N., 2003: Empirical attenuation relationship for Arias Intensity. - Earth. Eng. Struc. Dynam. 32(7): 1133-1155.

WOOD, H. \& NEWMAN, F., 1931: Modified Mercalli Intensity of 1931. - Bull. Seis. Soc. Am. 21:277-283. 